\title{
Review Article \\ Metal-Enhanced Fluorescence and FRET in Multilayer Core-Shell Nanoparticles
}

\author{
Jérémie Asselin, ${ }^{1}$ Mathieu L. Viger, ${ }^{1,2}$ and Denis Boudreau ${ }^{1}$ \\ ${ }^{1}$ Department of Chemistry and Center for Optics, Photonics and Lasers (COPL), Université Laval, Québec, QC, Canada G1K 7P4 \\ ${ }^{2}$ Laboratory for Bioresponsive Materials, University of California, San Diego, 9500 Gilman Dr. MC 0600, La Jolla, \\ CA 92093-0600, USA
}

Correspondence should be addressed to Denis Boudreau; denis.boudreau@chm.ulaval.ca

Received 16 February 2014; Accepted 28 April 2014; Published 9 June 2014

Academic Editor: Young-Seok Shon

Copyright (C) 2014 Jérémie Asselin et al. This is an open access article distributed under the Creative Commons Attribution License, which permits unrestricted use, distribution, and reproduction in any medium, provided the original work is properly cited.

\begin{abstract}
In recent years, various methods for the synthesis of fluorescent core-shell nanostructures were developed, optimized, and studied thoroughly in our research group. Metallic cores exhibiting plasmonic properties in the UV and visible regions of the electromagnetic spectrum were used to increase substantially the brightness and stability of organic fluorophores encapsulated in silica shells. Furthermore, the efficiency and range of Förster resonant energy transfer (FRET) between donor and acceptor molecules located in the vicinity of the metallic core was shown to be enhanced. Such multilayer nanoparticle architectures offer, in addition to the aforementioned advantages, excellent chemical and physical stability, solubility in aqueous media, low toxicity, and high detectability. In view of these enviable characteristics, a plethora of applications have been envisioned in biology, analytical chemistry, and medical diagnostics. In this paper, advances in the development of multilayer core-shell luminescent nanoparticle structures and selected applications to bioanalytical chemistry will be described.
\end{abstract}

\section{Introduction}

Fluorescence spectroscopy is a dominant research tool in many fields of science and technology, largely due to its high sensitivity, low cost, and ease of use, and has become massively popular in analytical and biological sciences, particularly in cellular and molecular imaging, flow cytometry, medical diagnostics, DNA sequencing, forensics, and genetic analysis [1-6]. To benefit from this high sensitivity, bright and stable luminescent labels are usually required. However, most commonly available organic dyes used for optical signaling suffer from some important limitations such as hydrophobicity, collisional quenching in aqueous media, low fluorescence quantum yield, and low resistance to photobleaching [7]. Therefore, the continued development of new fluorescence techniques relies on the investigation of novel strategies to overcome the limitations of current fluorescent probes.

In this regard, the remarkable optical properties displayed by metal nanostructures, in particular the coupling between the free electrons responsible for surface plasmon resonance and nearby fluorophores, can increase the local electrical field and enhance the excitation and emission rates and decrease the lifetimes of excited states [8-11]. This phenomenon, termed metal-enhanced fluorescence (MEF), is being investigated intensively and represents a powerful technology to increase the detection sensitivity of various biological assays [12-19]. Most of the studies performed to date involved twodimensional metallic surfaces, particularly those composed of silver islands, silver colloids (nanospheres, nanotriangles, nanorods), and even fractal-like silvered surfaces where glass or plastic slides were used as the primary substrates [20-26]. Several methods for the deposition of silver on substrates have been used, such as wet chemistry [25-27], electroplating [28], and lithographical methods [28]. These two-dimensional analytical devices are already shown as viable homogeneous protein sensors [29].

In comparison, much less attention has been given to systems where the metal is in the form of isolated nanoparticles (NPs) suspended in solution and coated with the fluorophore(s) of interest (Figure 1), due to the difficulties 
in achieving the required uniformity in geometry (size of metal core, metal-fluorophore spacing, fluorophore density, etc.) [30-39]. Nevertheless, despite their higher complexity, such systems offer significant benefits. The metal core of composite NPs confers them a strong plasmon resonance, and interaction with the surrounding fluorescent molecules greatly improves excitation and emission rates. Moreover, the reduction of the fluorophore's lifetime in the excited state increases photostability and detectability, and the ensuing reduction of self-quenching makes possible the incorporation of higher densities of fluorophore molecules around the metal core [40]. Controlled growth of a dielectric silica shell around the core ensures easy tunability of the dyemetal separation and of the resulting fluorescence enhancement [39]. Also, this shell protects the organic dyes against collisional quenching and can be functionalized to covalently bind target biomolecules or fluorophores or form electrostatic complexes with charged molecules [19, 41]. Finally, plasmonic enhancement in core-shell nanostructures improves the efficiency, range, and transfer rate of Förster resonance energy transfer (FRET) by increasing the strength of donor-acceptor interactions, which can in turn lead to the excitation by a single donor of several acceptors molecules over distances exceeding the natural range of FRET $[39,41]$.

Multilayer core-shell NPs present many of the features required of an ideal fluorescent probe, that is, high optical detection sensitivity, large excitation cross-section, excellent chemical and photophysical stability, low toxicity, high solubility in water, and easy conjugation to target biomolecules. Moreover, since almost any fluorophores can be incorporated in the silica shell, the absorption and emission spectra of the NPs are easily tunable from the UV to the near infrared (NIR) region, the latter being particularly valuable for studying biological samples where autofluorescence decreases progressively with increasing wavelength. Finally, the mobility of these NPs is an asset for probing the contents of extended sample volumes in biosensing applications or for functional cell imaging work.

\section{Preparation of Core-Shell Nanoparticles}

2.1. Synthesis of Metal Cores. The most popular method to prepare noble metal nanospheres dispersed in water is the well-known citrate reduction $[42,46]$, first introduced by Turkevich et al. [47]. The colloids are obtained by reducing metal salts (typically $\mathrm{AgNO}_{3}$ and $\mathrm{HAuCl}_{4}$ ) dissolved in boiling water with sodium citrate under vigorous stirring. By varying the concentration ratio between the metal salt and sodium citrate, the average particle diameter can be tuned over a wide range $(10-100 \mathrm{~nm})$. For gold colloids, this method leads to the formation of very uniform nanoparticles (Figure 2(a)), whereas the same procedure used for silver colloids results in the formation of larger particles with a higher polydispersity index (Figure 3(a)). However, the formation of uniform silver NPs can be obtained by adding the silver salt to a boiling sodium citrate solution. Buffering the reaction environment with sodium citrate allows better control of

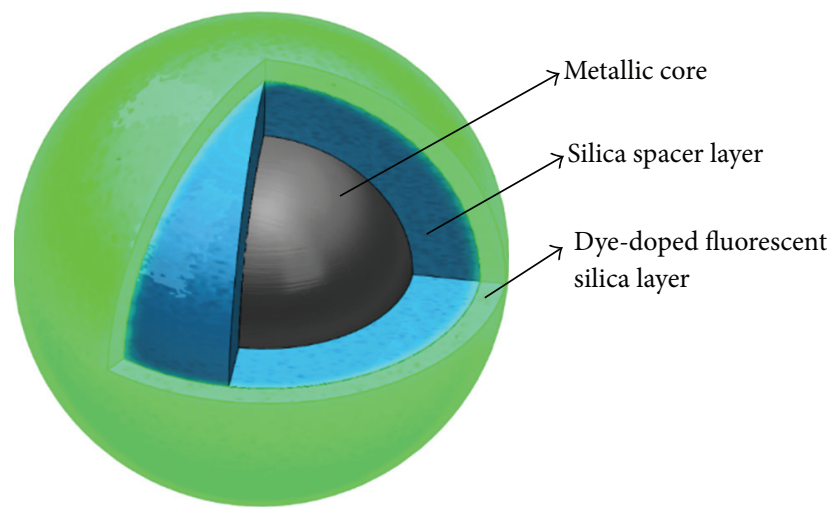

FIGURE 1: Schematic representation of the MEF-capable multilayer core-shell nanoparticle.

the shape and size distribution of the spherical silver NPs. By controlling the $\mathrm{pH}$ during the nucleation stage, one can prepare spherical silver NPs free of undesirable rod-like particles (Figure 3(b)) [48]. In similar conditions involving tannic acid as the reducing agent, lower polydispersity silver cores up to $118 \mathrm{~nm}$ in diameter have been obtained [49]. Borohydride reduction has also been used for the production of small noble metal NPs in aqueous media [50] and, notably, of small uniform Au NPs as seeds for the growth of larger spheres, nanorods, and nanoprisms [51].

Silver, platinum, indium, and bimetallic NPs can also be obtained with borohydride as the reducing agent $[42,52-54]$. For example, borohydride injection in a high-temperature indium salt and sodium citrate solution in diethylene glycol yields extremely spherical $\mathrm{In}^{0} \mathrm{NPs}$ [42]. Interestingly, group XIII metals such as aluminum and indium present a localized surface plasmon centered in the ultraviolet region, out of the normal range for noble metals particles. Therefore, by choosing the appropriate metal one can tune the core's optical properties for various applications. Since energy resonance of fluorescence with the plasmonic oscillation will induce an enhancement of different phenomena, the choice of fluorophores with a metallic core should overlap the plasmonic wavelengths with both excitation and emission spectra for optimal results [55].

2.2. Synthesis of Silica Coatings. The coating of metal colloids with silica is usually carried out using the so-called Stöber method $[45,56]$, which consists in hydrolyzing an alkoxide (e.g., tetraethoxysilane, TEOS) in a mixed solution of ammonia, alcohol, and water, and which can yield colloids with low polydispersity and nearly perfect core-shell structure (Figures 2(b) and 3(c)). The final thickness of the silica spacer shell can be controlled by varying the amount of alkoxide incorporated into the reaction mixture (Figure 3(c)). Another very useful method uses silane coupling agents as primers to form a monolayer of silanol $(-\mathrm{OH})$ groups on the surface of the metal NPs, followed by the deposition of a thin silica layer from a sodium silicate solution [57].

To obtain fluorescent NPs, a second, thinner dye-doped silica shell is grown over the silica spacer shell using 


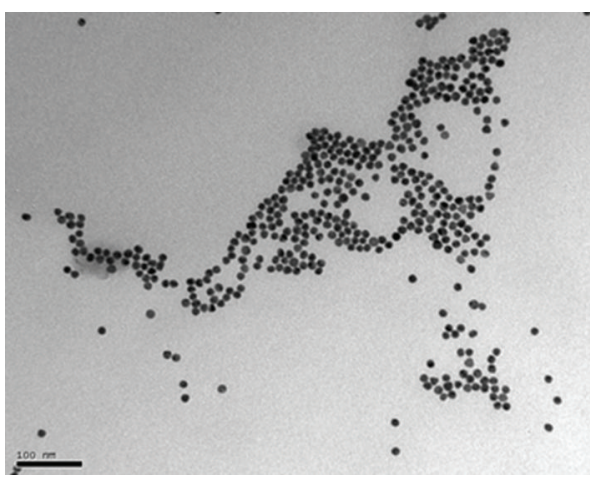

(a)

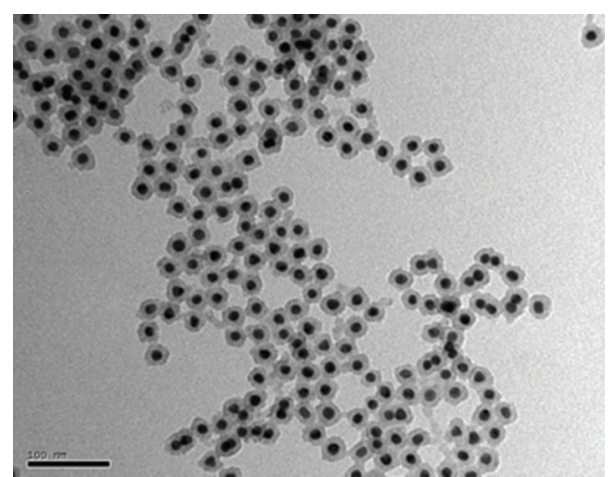

(b)

FIgURE 2: Transmission electron microphotographs of (a) bare and (b) silica-coated Au nanoparticles.

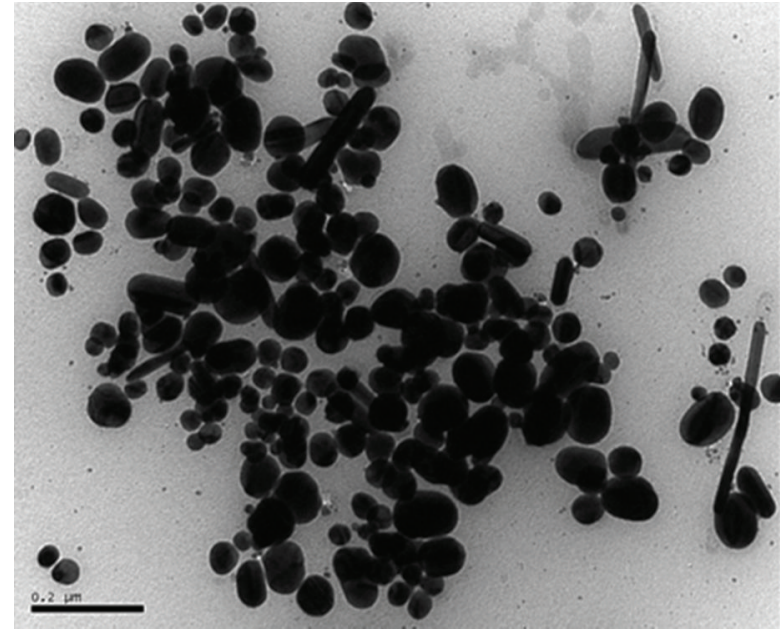

(a)

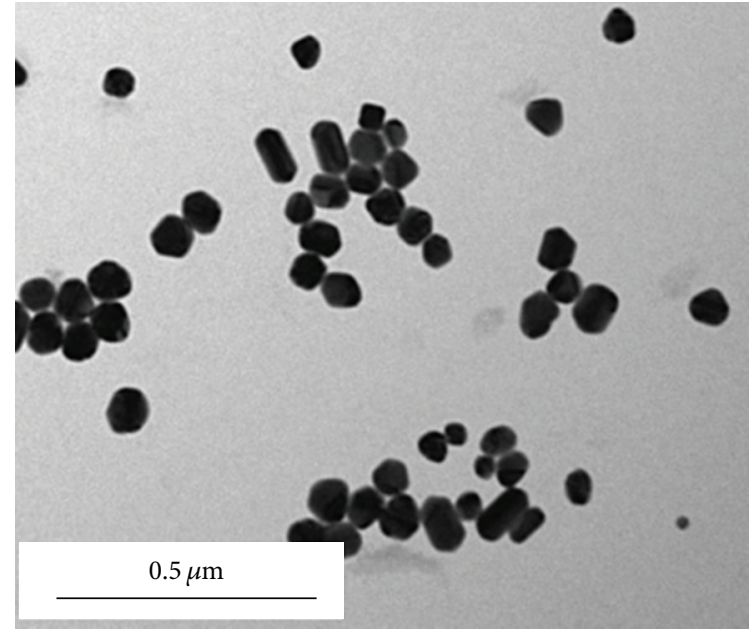

(b)

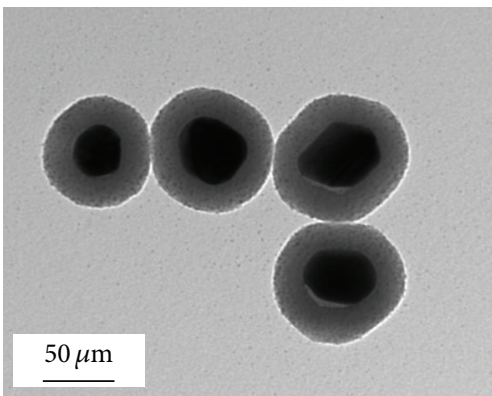

(c)

Figure 3: Transmission electron microphotographs of (a) high and (b) low polydispersity Ag nanoparticles and (c) silica-coated Ag nanoparticles with different coating thicknesses (left: $7 \pm 2 \mathrm{~nm}$, center: $13 \pm 2 \mathrm{~nm}$, right: $23 \pm 3 \mathrm{~nm}$ ). (Reprinted with permission from [39], Copyright 2009 American Chemical Society).

a dye-conjugated silane coupling agent to covalently bind the dye molecules inside the silica matrix, thereby preventing dye leakage and ensuring long-term luminescence stability. Typically, the reaction consists of mixing amine-reactive fluorescent dyes (e.g., isothiocyanate, succinimidyl ester, tetrafluorophenyl ester, sulfodichlorophenol ester, and sulfonyl chloride derivatives) with 3-(aminopropyl)triethoxysilane (APS) to form the reaction product APS-dye. For the most part, amine-reactive dyes are hydrophobic molecules and should be dissolved in high-quality, anhydrous ethanol, dimethylformamide (DMF), or dimethylsulfoxide (DMSO) (DMSO should be avoided with sulfonyl chlorides). The copolymerization of APS-dye with TEOS allows a variety of molecular dyes to be bound covalently to this outer silica shell. 


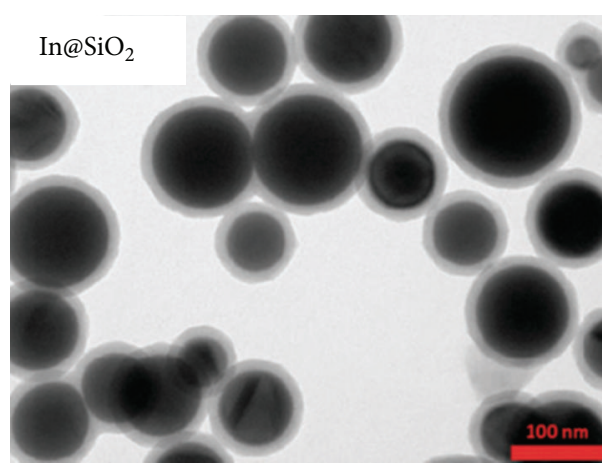

(a)

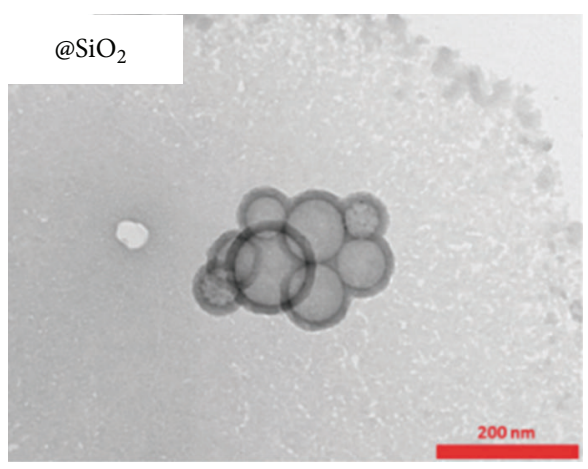

(b)

Figure 4: Transmission electron micrographs of core-shell structures and hollow silica nanoshells prepared from indium nanoparticles. (Reprinted with permission from [42], Copyright 2013 The Royal Society of Chemistry).

Spherical core-shell nanocomposites with a narrow size distribution can also be obtained by chemical reduction of metal salts within reverse micelles, followed by in situ hydrolysis and condensation of alkoxides in the microemulsion [58]. A reverse microemulsion (water in oil) is a singlephase system that consists of water, oil, and a surfactant, where water nanodroplets, stabilized by surfactant molecules and dispersed in the oil phase, serve as nanoreactors for the synthesis of NPs. This method ensures a narrow particle size distribution and avoids high temperature conditions that can degrade organic dyes, and the particle size and shape can be tuned easily by simple changes in the microemulsion conditions. Furthermore, this simple synthesis procedure is very fast, because the formation of metal cores, silica spacer, and dye-labeled outer silica shell occurs in the same microemulsion; in comparison, other approaches used in the preparation of this type of core-shell particles are more timeconsuming, requiring either more synthesis and centrifugation steps or longer reaction times.

\subsection{Surface Functionalization. Biofunctionalization through} surface modification of the as-prepared fluorescent coreshell NPs can be performed in several ways [59]. The modification of silica shells to provide sites for coupling affinity ligands can be done through covalent derivatization of the surface with a functionalized silane linker. Reagents containing alkoxysilane groups condense with the silanol groups on silica shell surfaces to form stable siloxane linkages. Choosing the appropriate silane linker allows tailored surface modification and subsequent coupling of biomolecules. For instance, reaction with APS coats the surface of the coreshell NPs with primary amino groups for conjugation with aldehyde or carboxylate-linked compounds via reductive amination or carbodiimide-mediated coupling with EDC (1ethyl-3-(3-dimethylaminopropyl)carbodiimide). The amineactivated surfaces can also be modified more conveniently to contain carboxylate groups via a simple reaction with succinic anhydride. Carboxylated core-shell NPs may then be coupled with common amine-containing ligands, such as oligonucleotides and proteins, also using the standard carbodiimide reaction with EDC. Other useful silanation reagents are (3-mercaptopropyl) trimethoxysilane, which provides thiol $(-\mathrm{SH})$ groups compatible with maleimidelinked compounds, and (3-glycidoxypropyl)triethoxysilane, which contains a reactive epoxy group that can be used to conjugate thiol-, amine-, or hydroxyl-containing ligands. The binding capacity of biofunctionalized core-shell NPs can be determined experimentally by measuring the amount of dye-labeled target molecules left in the supernatant after the conjugation step with the NPs.

\section{Spectroscopic Characterization}

To evaluate the photophysical effects of the plasmonic core, control samples, that is, hollow silica nanoshells, may be prepared from fluorescent core-shell NPs by dissolving the metal cores with either cyanide or chloride ions, taking advantage of the porous nature of the silica shell matrix (Figure 4) [33, 57]. It should be noted that the fluorophores incorporated into the silica shell by covalent bonding are not displaced by this etching process. For other metallic cores (indium, platinum, etc.), more rigorous etching conditions require the use of nitric acid for the dissolution process, and care must be taken not to disrupt the organic binding [42]. These hollow nanoshells are believed to provide a more accurate determination of the fluorescence enhancement factor than solid silica NPs, because the latter and silica shells are grown by different synthetic routes (i.e., homogenous and heterogeneous nucleation processes, resp.) and the labeling efficiencies are likely to be different for the two types of nanoparticles. Etching the metal cores results in a pronounced drop in fluorescence signal simultaneously with the disappearance of the plasmonic band (Figure 5). The extinction spectra of eosin- $5^{\prime}$-isothiocyanate (EiTC-)doped $\mathrm{Ag} @ \mathrm{SiO}_{2}$ nanocomposites and of hollow silica nanoshells containing different quantities of EiTC are shown in Figure 6. The extinction spectrum for the core-shell NPs (top curve) is dominated by plasmonic absorption from the silver cores (centered at $420 \mathrm{~nm}$ ), whereas the complete dissolution of the metal cores to form hollow silica nanoshells is confirmed by the disappearance of this plasmon band from the spectrum (bottom curves). Furthermore, the absorption signal 


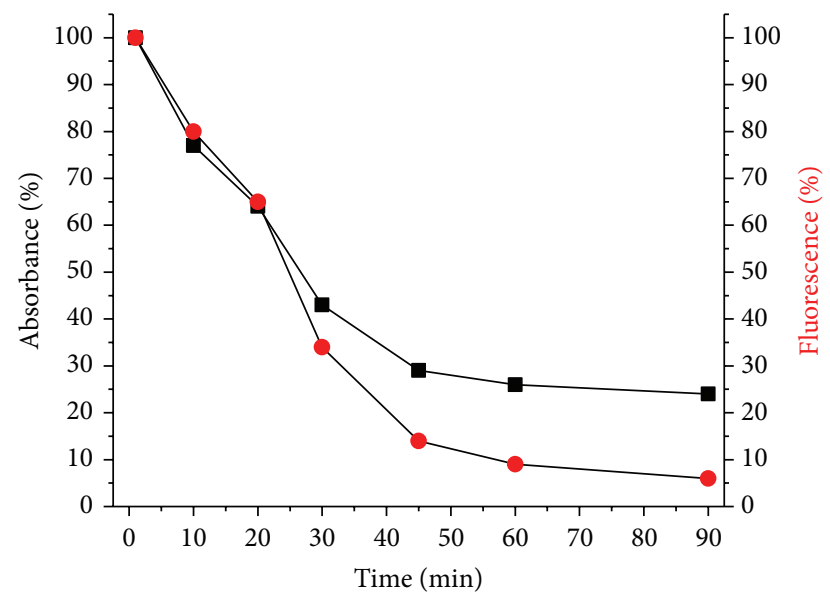

FIgURE 5: Simultaneous decrease of plasmon absorption (black squares) and fluorescence signal intensities (red circles) upon etching of the metal core from eosin- $5^{\prime}$-isothiocyanate (EiTC-)doped $\mathrm{Ag} @ \mathrm{SiO}_{2}$ core-shell nanocomposites.

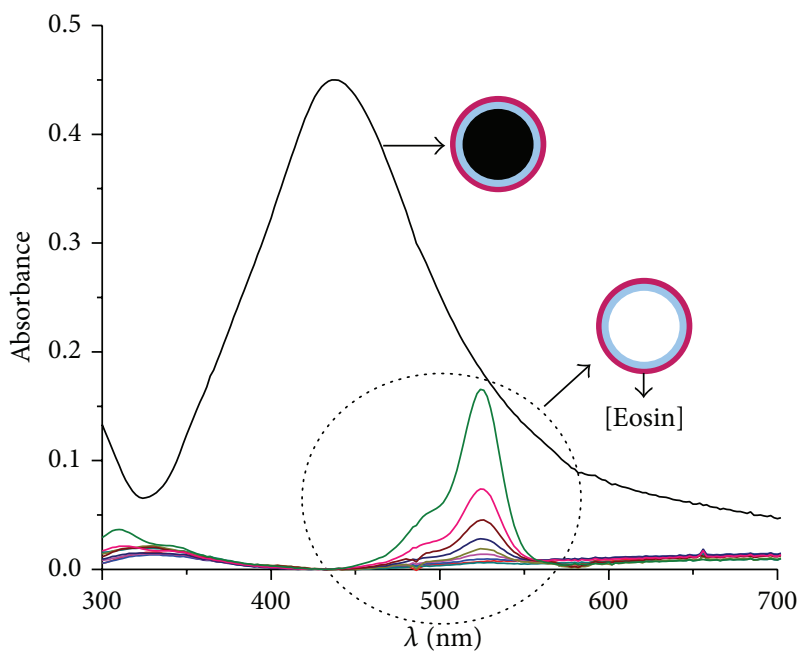

FIGURE 6: Extinction spectra of $\mathrm{Ag} @ \mathrm{SiO}_{2} @ \mathrm{SiO}_{2}$ + EiTC NPs (upper curve) and corresponding hollow silica nanoshells (lower curves). Silica spacer shell thickness was $7 \pm 2 \mathrm{~nm}$, concentration of EiTC was $4 \mathrm{mM}$ in the core-shell nanoparticles (upper curve) and from 0 to $87 \mathrm{mM}$ in hollow silica shells (lower curves). (Reprinted with permission from [39], Copyright 2009 American Chemical Society).

intensity of EiTC at $520 \mathrm{~nm}$ follows the amount of EiTClabeled silane bound to the silica material, indicating that the controlled incorporation of dye in core-shell NPs is effective and that covalent binding resists the etching process.

Finally, the amount of fluorophore incorporated in the various core-shell NPs can be determined by dissolving the silica shells in aqueous sodium hydroxide, which breaks down the silica network and releases the dye molecules which can be measured by standard fluorimetry. The amount of nanoparticles can be determined by dissolving the metal cores in chloride, cyanide, or nitric acid and measuring the resulting aqueous solution by atomic absorption spectrometry.

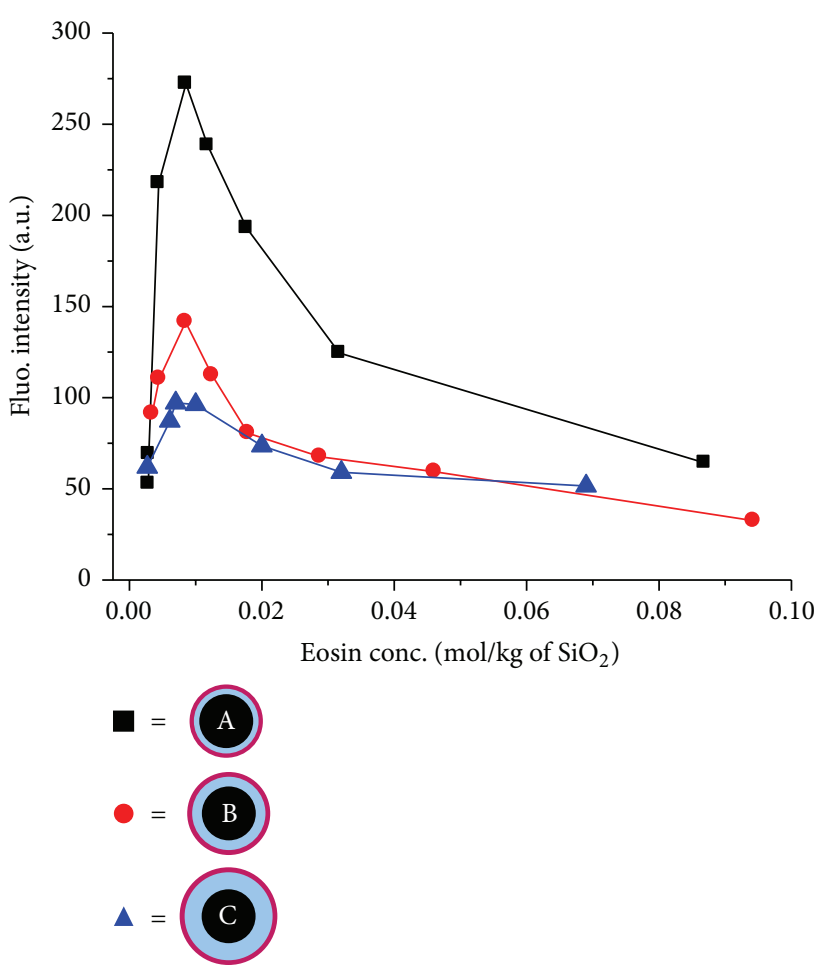

FIGURE 7: Influence of metal core proximity and dye concentration on the fluorescence intensity of $\mathrm{Ag@SiO} @_{2} @ \mathrm{SiO}_{2}+$ eosin nanocomposites. Three different spacer thicknesses were studied ((A) $7 \pm$ $2 \mathrm{~nm}$; (B) $13 \pm 2 \mathrm{~nm}$; (C) $23 \pm 3 \mathrm{~nm}$ ). (Reprinted with permission from [39], Copyright 2009 American Chemical Society).

\section{Dependence of Metal-Enhanced Fluorescence on Distance}

The use of silica spacer shells with uniform and tunable thickness around the metal cores allows precise optimization of fluorescence enhancement. The influence of shell thickness on the extent of MEF is shown in Figure 8 for $\mathrm{Ag@SiO}{ }_{2} @ \mathrm{SiO}_{2}+\mathrm{EiTC}$ samples (core size of $\sim 45 \mathrm{~nm}$ ), where the luminescence intensity is shown to drop rapidly when the spacer thickness is increased from 7 to $23 \mathrm{~nm}$. The highest luminescence intensity was observed for the NPs with the thinnest spacer shell (7 $\pm 2 \mathrm{~nm}$; Figure 7$)$, which is in accordance with previous reports $[30,32,33,60]$.

As described in Section 2, one can dissolve the core from core-shell NPs with chloride (for $\mathrm{Ag}$ ), cyanide ions ( $\mathrm{Au}, \mathrm{Ag}$ ), or nitric acid (In) to confirm the influence of the metal cores on fluorescence emission. These reagents have advantages and disadvantages associated with their use. Acidification with $\mathrm{HNO}_{3}$ can influence the aggregation of silica surfaces [61], whereas dissolution using cyanide is faster than with chloride (a few hours versus overnight to $24 \mathrm{~h}$ ) but suffers from well-known fluorescence quenching effects. Thus, to measure the absolute enhancement factor, one needs to compensate for the effect of quenching, that is, quantifying the influence of quenching on the fluorescence signal of the dye and normalize the fluorescence intensity accordingly. Alternatively, an additional centrifugation step 


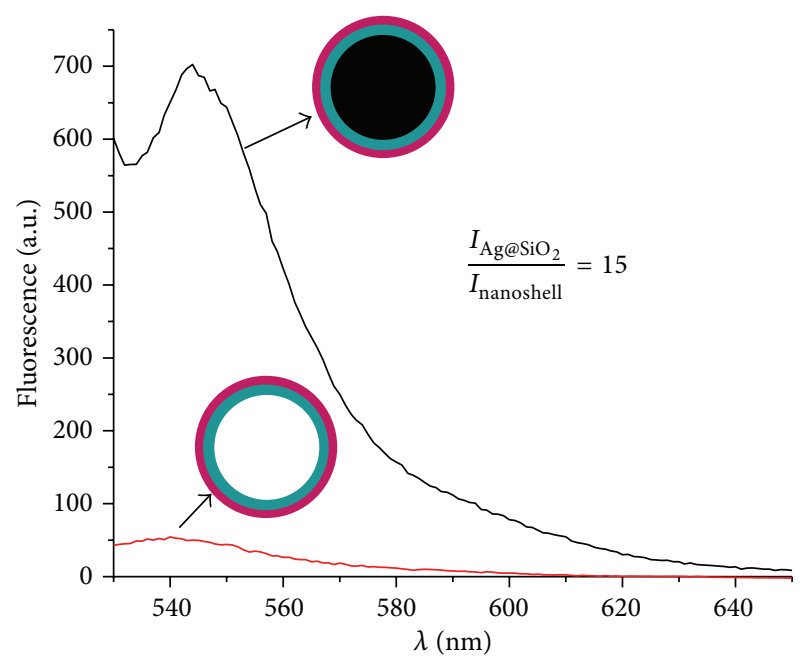

(a)

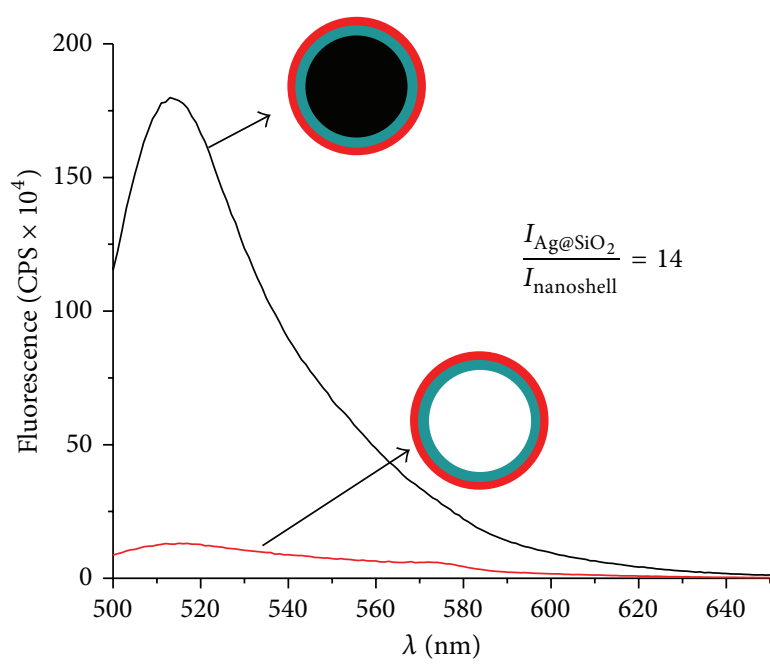

(b)

FIGURE 8: MEF factors for eosin (a) and fluorescein (b) determined from the emission spectra of core-shell NPs and their corresponding nanoshells. The thickness of the silica spacer is $\sim 7 \mathrm{~nm}$ for both samples. (Reprinted with permission (a) from [38], Copyright 2009 and (b) from [41], Copyright 2011, American Chemical Society).

may be used to remove cyanide ions before the measurement of fluorescence; however, the risk of losing hollow silica nanoshells during centrifugation is high because their small mass requires very high sedimentation rates, which are difficult to achieve with conventional centrifuges. Interestingly, the use of chloride (which is a poorer quenching agent than cyanide) to etch the metal cores, while being more timeconsuming, allows MEF factors to be determined without centrifugation.

Comparing the fluorescence spectra from $\mathrm{Ag} @ \mathrm{SiO}_{2} @ \mathrm{SiO}_{2}+$ EiTC NPs ( 45 nm core diameter $)$ with those from their corresponding coreless control samples has shown a 15 -fold signal intensity in the presence of the metal core for a dye-to-metal separation of $7 \pm 2 \mathrm{~nm}$ (Figure 8(a)). Lower enhancement factors of 5 and 2 were measured for spacer thicknesses of $13 \pm 2$ and $23 \pm 3 \mathrm{~nm}$, respectively. Similarly, a 14 -fold fluorescence enhancement factor was measured for $\mathrm{Ag} @ \mathrm{SiO}_{2} @ \mathrm{SiO}_{2}+$ FiTC NPs with a similar spacer shell thickness of $\sim 7 \mathrm{~nm}$ (Figure 8(b)).

Depending on the metal used for the plasmonic core, a metal oxide layer may form on the surface which does not contribute to the enhancement of fluorescence. In the case of indium, for example, a more realistic core-shell structure would be In@InO $\mathrm{In}_{x} @ \mathrm{SiO}_{2}$ with an oxide layer $~ 5 \mathrm{~nm}$ in thickness $[42,62]$. For such nanostructures, both the $\mathrm{MO}_{x}$ and $\mathrm{SiO}_{2}$ layers have to be considered in the calculation of the core-fluorophore distance.

The lifetime of a fluorophore depends on its radiative decay rate; therefore, the core-induced increase in fluorescence noted previously for dye-doped $\mathrm{Ag@SiO}$ NPs should be accompanied by a shortening of the excitedstate lifetime. Fluorescence lifetime measurements for core-shell NPs, their corresponding hollow silica nanoshells, and the fluorophore free in solution show that the core-shell NPs systematically have a shorter lifetime. For example, we measured an average fluorescence lifetime of $0.228 \mathrm{~ns}$ for eosin in core-shell NPs with a $7 \pm 2 \mathrm{~nm}$ thick spacer, which represents a distinctive reduction in lifetime over that of eosin bound to bare silica and free in solution [39]. Similarly, a reduction in excited-state lifetime from 1.72 to 0.03 ns was measured for FiTC in $\mathrm{Ag} @ \mathrm{SiO}_{2} @ \mathrm{SiO}_{2}+$ FiTC NPs of comparable core size and spacer thickness as compared with corresponding hollow silica nanoshells [41]. These observations are manifestations of the MEF phenomenon, in which metal-fluorophore interactions lead to an increase in the quantum yield of the fluorophore and a decrease in its lifetime. Because photodegradation occurs while the fluorophore is in the excited state, a decrease in fluorescence lifetime should result in increased photostability. In other words, dye molecules located closer to the metal surface can undergo more excitationrelaxation cycles prior to photobleaching, resulting in a substantial increase in the number of detectable photons [63].

The proximity of fluorophores to metallic particles enables the modification of radiative decay rates. It is possible to determine the radiative rate enhancement from experimentally measured changes in lifetime. To do so, one must combine and rearrange the mathematical expressions defining the quantum yield and lifetime of a fluorophore free in solution and close to metal particles. For a fluorophore free in solution, the quantum yield $(\Phi)$ and lifetime $(\tau)$ can be expressed as [7]

$$
\begin{aligned}
& \Phi=\frac{\Gamma}{\Gamma+k_{\mathrm{nr}}}, \\
& \tau=\frac{1}{\Gamma+k_{\mathrm{nr}}},
\end{aligned}
$$




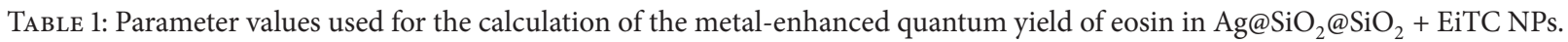

\begin{tabular}{|c|c|c|c|c|c|c|}
\hline$\Phi_{0}$ & $\tau_{\text {eosin in soln }}(\mathrm{ns})$ & $\tau_{M}(\mathrm{~ns})$ & $k_{\mathrm{nr}}\left(\mathrm{s}^{-1}\right)$ & $\Gamma\left(\mathrm{s}^{-1}\right)$ & $\gamma \Gamma\left(\right.$ or $\left.\Gamma_{M}\right)\left(s^{-1}\right)$ & $\Phi_{M}$ \\
\hline 0.57 & 2.83 & 0.228 & $1.52 \times 10^{8}$ & $2.01 \times 10^{8}$ & $4.23 \times 10^{9}$ & 0.97 \\
\hline
\end{tabular}

Definition of headers: $\Phi_{0}$ is the unmodified quantum yield of eosin in solution, $\tau_{\mathrm{M}}$ is the metal-enhanced lifetime of eosin in core-shell NPs, $k_{\mathrm{nr}}$ is the nonradiative decay rate, $\Gamma$ is the radiative decay of eosin in solution, $\gamma \Gamma$ (or $\Gamma_{\mathrm{M}}$ ) represents the metal-enhanced radiative decay rate of eosin in core-shell NPs, and $\Phi_{M}$ is the metal-enhanced quantum yield of eosin in core-shell NPs.

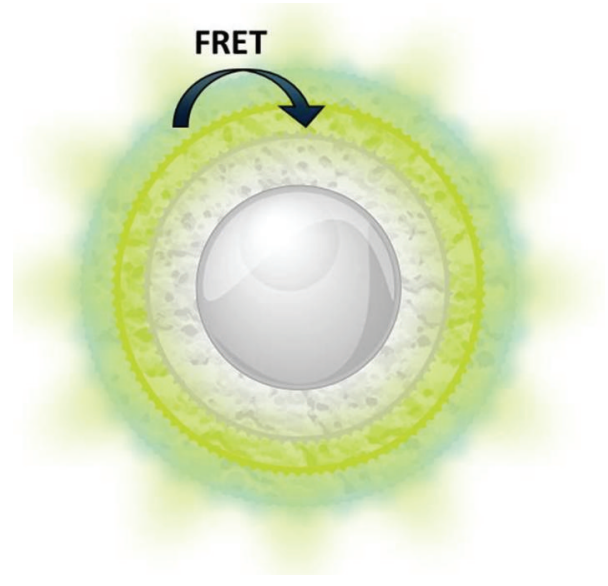

Silver core

Spacer silica layer

1st fluorescent silica layer (acceptor)

2nd fluorescent silica layer (donor)

FIGURE 9: Multilayer nanoarchitecture featuring a metal core surrounded by a silica spacer layer and fluorescent silica layers containing FRET donor and acceptor molecules. (Reprinted with permission from [39], Copyright 2009 American Chemical Society).

where $\Gamma$ and $k_{\mathrm{nr}}$ are the radiative and nonradiative decay rates, respectively. When a fluorophore is perturbed by a metallic particle, the plasmonic coupling causes an increase in the molecular radiative decay rate by a factor of $\gamma$, and the metal-enhanced quantum yield $\left(\Phi_{M}\right)$ and lifetime $\left(\tau_{M}\right)$ values become $[30,41]$

$$
\begin{gathered}
\Phi_{M}=\frac{\gamma \Gamma}{\gamma \Gamma+k_{\mathrm{nr}}}, \\
\tau_{M}=\frac{1}{\gamma \Gamma+k_{\mathrm{nr}}},
\end{gathered}
$$

where $\gamma \Gamma$ represents the effective radiative decay rate. The radiative rate $\left(\Gamma_{M}=\gamma \Gamma\right)$ and quantum yield $\left(\Phi_{M}\right)$ of coreshell NPs can be determined by using the equations above

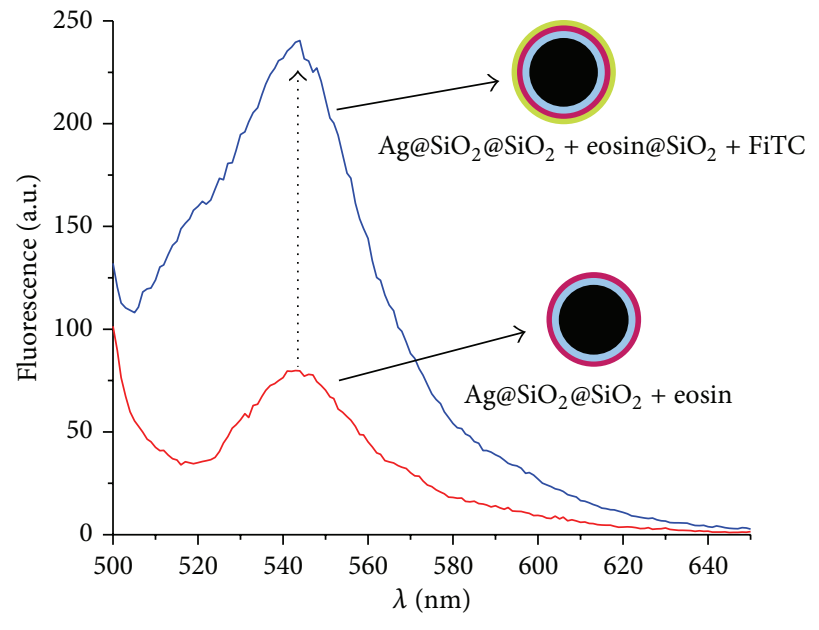

FIGURE 10: Fluorescence spectra recorded for donor + acceptor multishell and acceptor-only core-shell nanocomposites; [FiTC] = $7.2 \mathrm{mM}$ and $[$ EiTC] $=8.5 \mathrm{mM}$. (Reprinted with permission from [39], Copyright 2009 American Chemical Society).

and measuring the change in fluorescence lifetime caused by plasmonic coupling, based on the assumption that the nonradiative decay rate $k_{\mathrm{nr}}$ is unaffected by the presence of the metal core (as stated above, the total nonradiative decay rate of a fluorophore near a metal surface is known to be negligible for distances over $4 \mathrm{~nm}$, versus the silica spacer thickness $\geq 7 \mathrm{~nm}$ used in these studies). A value of $97 \%$ was thus calculated for the metal-enhanced quantum yield $\Phi_{M}$ of eosinin $\mathrm{Ag} @ \mathrm{SiO}_{2} @ \mathrm{SiO}_{2}+\mathrm{EiTC}$, as compared to 57\% for the eosin molecules free in aqueous solution (Table 1).

The quantum efficiency of most organic dyes is strongly impacted by self-quenching when their relative concentrations exceed $\sim 10^{-3} \mathrm{M}$ which results from the higher probability of close-range interactions between aggregated dye molecules and longer range intermolecular energy transfer processes (e.g., homotransfer between separated dye molecules and transfer between excited monomers and neighboring quenched aggregates) [64]. While selfquenching directly affects the maximum fluorophore concentration usable in dye-doped silica materials, it was reported by us [40] and others [43] that its severity among other labeled silica matrices can be reduced by the proximity of metallic particles; a phenomenon attributed to a strong decrease in nonradiative decay rates, that is, a reduction in energy transfer between dye molecules. 

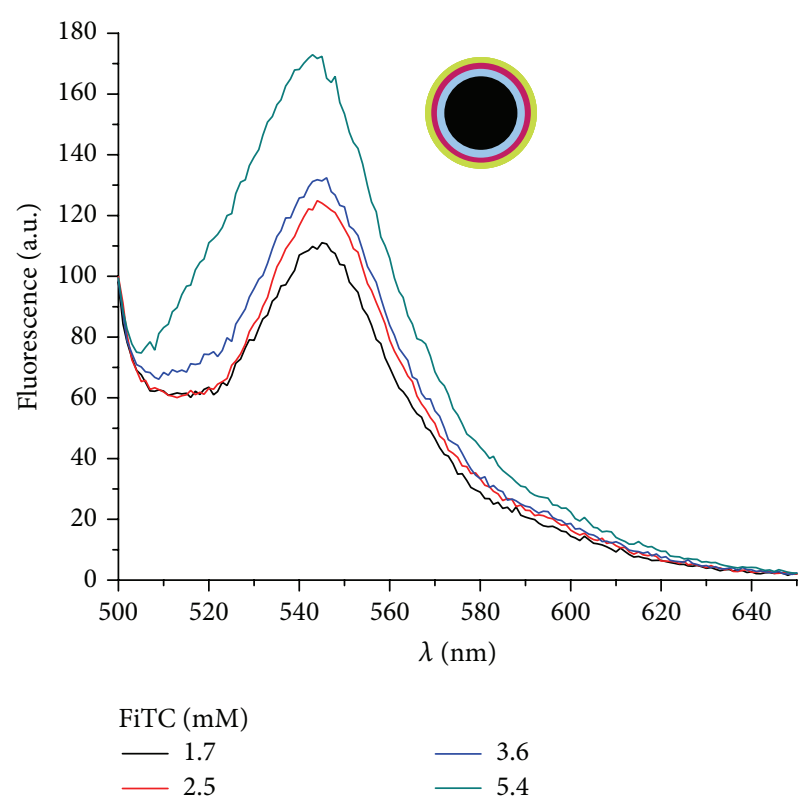

(a)
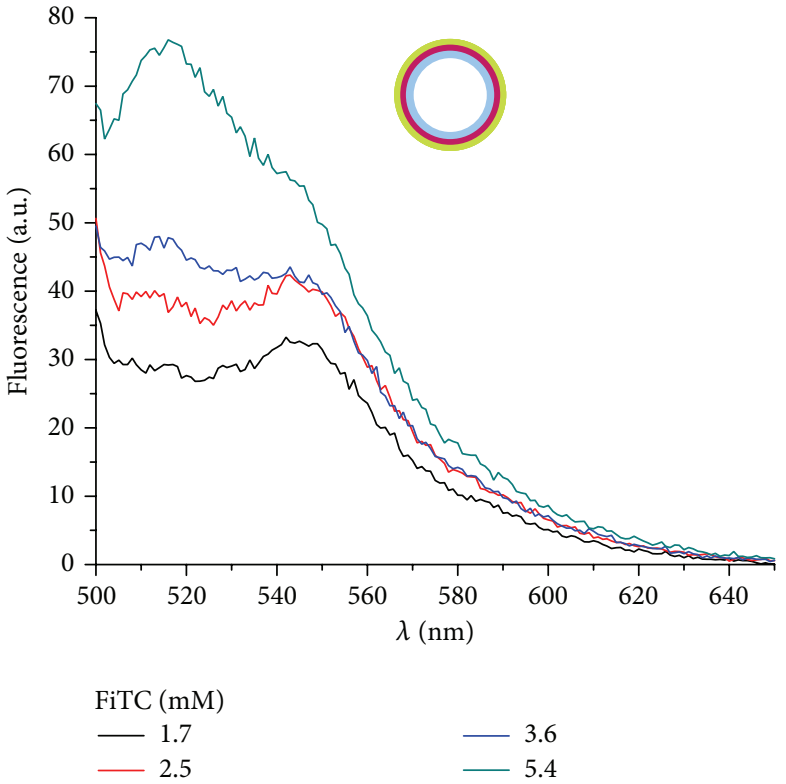

(b)

FIGURE 11: Fluorescence spectra recorded for donor + acceptor multishell nanoparticles (a) and corresponding hollow silica nanoshells (b) in which the concentration of FiTC (donor) was increased from 1.7 to $5.4 \mathrm{mM}$, while the concentration of EiTC (acceptor) was kept constant at 12 mM. (Reprinted with permission from [39], Copyright 2009 American Chemical Society).

\section{Metal-Enhanced Förster Resonant Energy Transfer}

In addition to the enhancement of fluorescence, metal NPs are also capable of influencing FRET between two molecules. Recent theoretical and experimental studies have shown that positioning donor-acceptor pairs close to metal NPs results in an enhancement of transfer rate, along with an increase in the efficiency and range of FRET $[36,39,65,66]$. We used the layer-by-layer procedure described in Section 2 to deposit a thin $(\sim 3 \mathrm{~nm})$ silica layer containing a donor or acceptor dye over Ag@SiO $@_{2} @ \mathrm{SiO}_{2}+$ dye labeled with a complementary dye (Figure 9). Keeping both fluorescent shells at a minimal thickness and in direct contact maximizes the number of donors and acceptors within the Förster range achievable with these core-shell nanostructures. Given the strong dependence of MEF and distance to the metal core (Figure 7), the efficiency of MEF for a given dye can drop significantly across a distance as small as the thickness of the dye-labeled layer (i.e., $\sim 3 \mathrm{~nm}$ ). Therefore, FRET enhancement will be greater by placing the dye with the lowest quantum yield (either the donor or the acceptor) closest to the metal core. For example, while studying the FRET pair eosin/fluorescein, we chose to position the eosin dye, which has a lower quantum yield than fluorescein, closer to the metal core.

We recorded fluorescence spectra for $\mathrm{Ag} @ \mathrm{SiO}_{2} @ \mathrm{SiO}_{2}+$ eosin@ $\mathrm{SiO}_{2}+$ fluorescein (eosin being the acceptor and fluorescein the donor) and acceptor-only Ag@SiO ${ }_{2} @ \mathrm{SiO}_{2}+$ eosin (i.e., before coating with the donor) nanocomposites, and the results show that the addition of the donor-labeled layer provides a $\times 3$ increase in the emission intensity of the acceptor at $545 \mathrm{~nm}$, despite the fact that the acceptor concentration is the same in both types of nanocomposites (Figure 10). Only a slight contribution of donor emission at $520 \mathrm{~nm}$ is perceivable in the spectrum of the donor + acceptor multishell sample. The high acceptor/donor emission ratio observed with these nanocomposites indicates the presence of efficient resonant energy transfer from the donor to the acceptor.

The influence of plasmonic coupling to the metal core on the extent of FRET in such nanocomposites can be studied by comparing the fluorescence spectra recorded before and after dissolution of the metal core, for a fixed concentration of acceptor and various donor concentrations (Figure 11). It was found that, in the presence of the silver core, the emission intensity of the eosin acceptor at $545 \mathrm{~nm}$ increases as the FiTC donor concentration in the nanoparticles is increased, and that donor emission at $520 \mathrm{~nm}$ is barely perceptible (Figure 11(a)). On the other hand, increasing the amount of donor molecules on the surface of coreless nanoparticles results mainly in the increase of donor emission intensity (Figure 11(b)), a clear indication that FRET efficiency between fluorescein and eosin is relatively poor in coreless multishell NPs, despite the relative proximity of the fluorescein and eosin layers. Fluorescence spectra were also acquired and compiled for core-shell NPs and their corresponding nanoshells as fluorescein concentration was kept constant and eosin concentration was increased progressively (Figure 12). The fluorescence intensity of eosin at $545 \mathrm{~nm}$ is shown to increase with concentration for both core-shell and coreless nanoparticles, but the fluorescence signal is considerably stronger in presence of the silver core. 

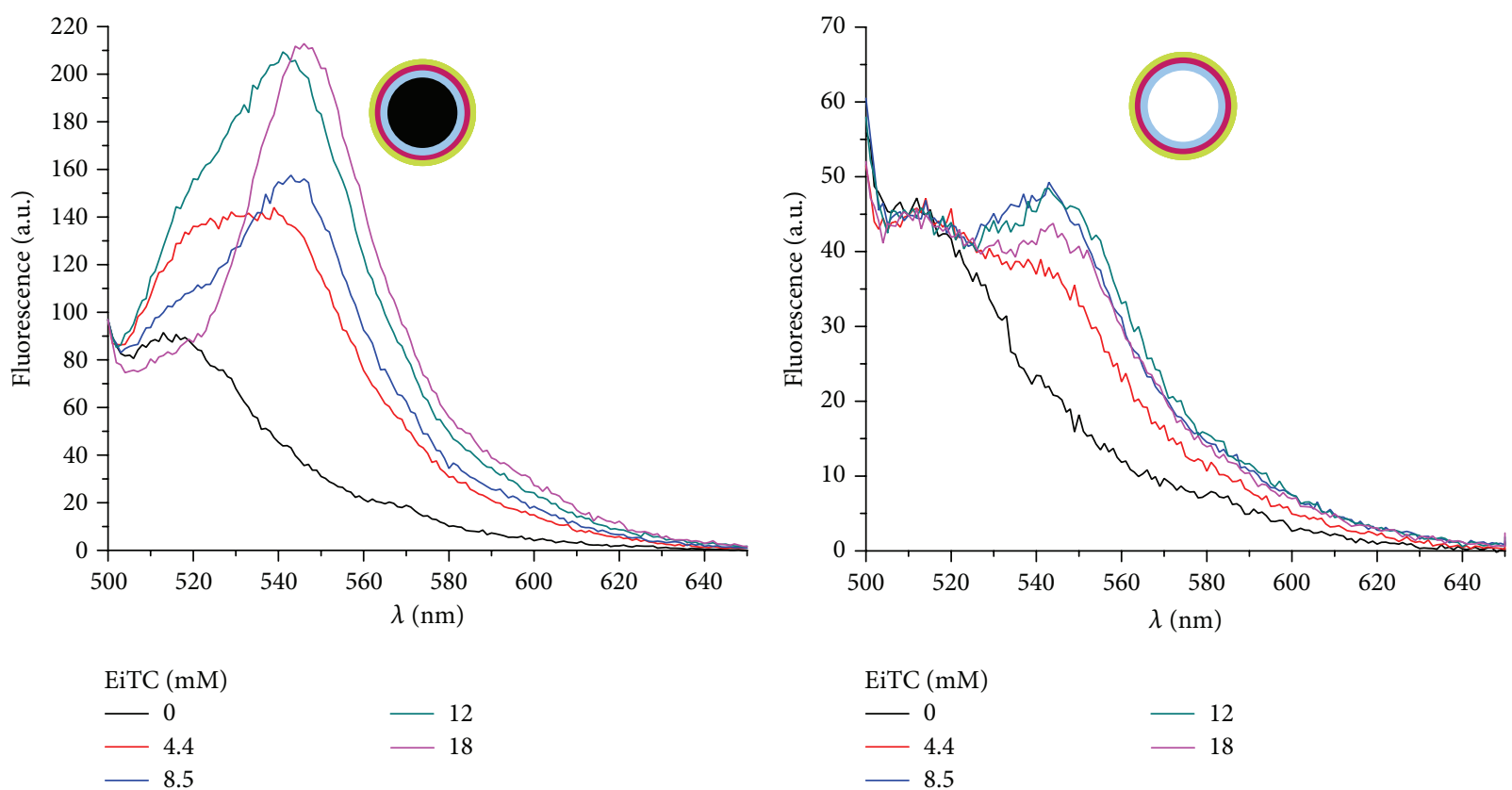

(a)

(b)

FIGURE 12: Fluorescence spectra recorded for donor + acceptor multishell nanoparticles (a) and corresponding hollow silica nanoshells (b) in which the concentration of EiTC (acceptor) was increased from 0 to $18 \mathrm{mM}$, while the concentration of FiTC (donor) was kept constant at $10 \mathrm{mM}$.

TABLE 2: Fluorescence lifetime data for CCP in solution and as CCP/SiO ${ }_{2}, \mathrm{CCP} / \mathrm{Ag} @ \mathrm{SiO}_{2}$, and CCP/Ag@SiO $@ \mathrm{SiO}_{2}+\mathrm{FiTC}_{2}$ nanoparticles (reprinted with permission from [40], Copyright 2011 American Chemical Society).

\begin{tabular}{|c|c|c|c|c|c|c|}
\hline & $\begin{array}{c}\tau_{1} \\
\text { (ns) }\end{array}$ & $\begin{array}{l}A_{1} \\
(\%)\end{array}$ & $\begin{array}{c}\tau_{2} \\
(\mathrm{~ns})\end{array}$ & $\begin{array}{l}A_{2} \\
(\%)\end{array}$ & $\begin{array}{c}\tau_{\mathrm{av}} \\
(\mathrm{ns})\end{array}$ & $\chi^{2}$ \\
\hline Free in solution $^{\mathrm{a}}$ & 0.188 & 100 & & & 0.188 & 1.008 \\
\hline $\mathrm{CCP} / \mathrm{SiO}_{2}{ }^{\mathrm{b}}$ & 0.119 & 100 & & & 0.119 & 1.003 \\
\hline $\mathrm{CCP} / \mathrm{Ag} @ \mathrm{SiO}_{2}{ }^{\mathrm{b}}$ & 0.028 & 100 & & & 0.028 & 1.009 \\
\hline $\mathrm{CCP} / \mathrm{Ag} @ \mathrm{SiO}_{2} @ \mathrm{SiO}_{2}+\mathrm{FiTC}^{\mathrm{b}}$ & 0.014 & 99.98 & 0.81 & 0.02 & 0.014 & 1.020 \\
\hline
\end{tabular}

${ }^{\mathrm{a}} \lambda_{\mathrm{ex}}=405 \mathrm{~nm} ; \lambda_{\mathrm{em}}=530 \mathrm{~nm} ;{ }^{\mathrm{b}} \lambda_{\mathrm{ex}}=405 \mathrm{~nm} ; \lambda_{\mathrm{em}}=490 \mathrm{~nm}$.

Definition of headers: $\tau_{1}, A_{1}, \tau_{2}, A_{2}$ are the lifetime and amplitude values for the first and second fitting components, respectively; $\tau_{\mathrm{av}}$ is the averaged lifetime value and $\chi^{2}$ is the "goodness of fit" parameter.

These experiments (Figures 11 and 12) show unequivocally an enhancement of FRET efficiency for donor and acceptor fluorophores located near silver particles.

Plasmon-enhanced FRET was also studied using a coreshell system in which a fluorescent cationic conjugated polymer (CCP) was electrostatically adsorbed on fluorescent core-shell NPs to form CCP/Ag@SiO ${ }_{2} @ \mathrm{SiO}_{2}+$ fluorescein nanocomposites (Figure 13) [41]. The electrostatic complexation of the CCP onto core-shell NPs was ensured by the positive charge of the CCP and the negative charge of the silica surface. Fluorescein was chosen as the energy acceptor because of its excellent spectral overlap with the emission band of the CCP donor. Figure 14 shows the fluorescence spectra recorded for the donor-acceptor nanocomposites and their corresponding donor-only and acceptor-only coreshell NPs. Upon excitation at $400 \mathrm{~nm}$, the donor-acceptor nanocomposites displayed a reduction in donor emission intensity and a 5-fold increase in acceptor emission intensity. These results suggest the presence of efficient energy transfer from the surface-bound CCP to the encapsulated fluorescein.

In order to confirm the influence of MEF on FRET in this system, fluorescence decay curves of fluorescein were recorded for core-shell NPs and their corresponding silica particles for a fixed concentration of CCP (donor) molecules and fluorescein (acceptor) concentrations (Table 2). The results show that the increase in resonant energy transfer described above is accompanied, as predicted by theory, by a decrease in the excited state lifetime of the donor; that is, the CCP lifetime decreases from 28 to 14 ps as fluorescein concentration is increased, in the presence of the silver cores. In the case of the doped silica samples, intermolecular distances are still too large to induce significant RET without the assistance of MEF, which is consistent with the lesser relative diminution in lifetime, that is, from 0.188 to $0.119 \mathrm{~ns}$, 


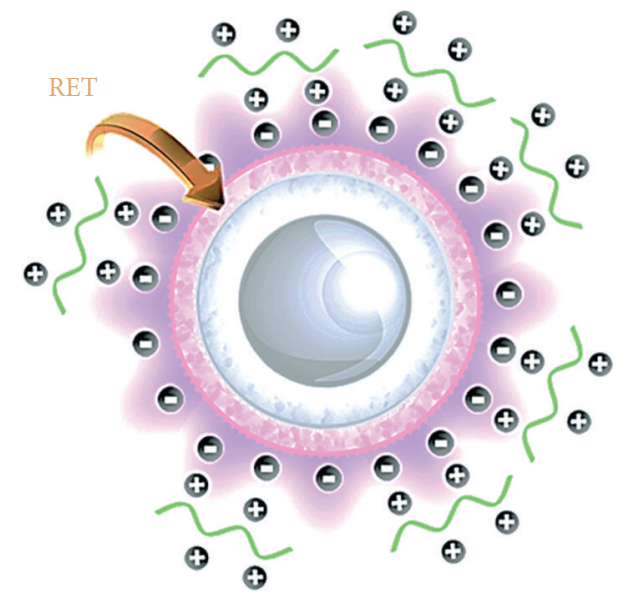

Silver core

Spacer silica layer

Fluorescent silica layer (acceptor)

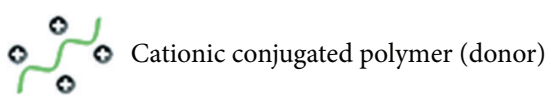

FIGURE 13: Multilayer nanoarchitecture featuring a metal core surrounded by a silica spacer layer, a fluorescent silica layer containing acceptor molecules, and cationic conjugated polymers absorbed on the outer fluorescent silica shell through electrostatic interactions. (Reprinted with permission from [41], Copyright 2011 American Chemical Society).

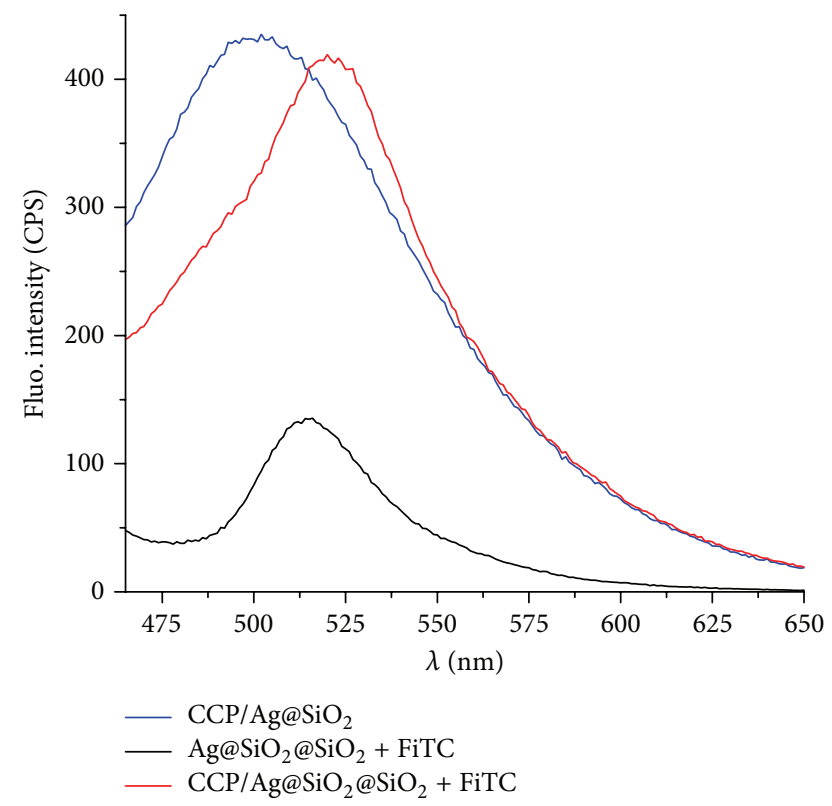

FIGURE 14: Comparison of fluorescence emission spectra recorded for CCP/Ag@SiO 2 (donor only), Ag@SiO $\mathrm{SSiO}_{2}$ + FiTC (acceptor only), and CCP/Ag@SiO $@ \mathrm{SiO}_{2}+$ FiTC (donor-acceptor) nanocomposites. (Reprinted with permission from [41], Copyright 2011 American Chemical Society). and the absence of significant FRET in coreless samples (Figures 11-12).

The lifetime of the donor alone $\left(\tau_{D}\right)$ and in the presence of the acceptor $\left(\tau_{D A}\right)$ is related to the FRET efficiency $(E)$ by the following equation [7]:

$$
E=1-\frac{\tau_{D A}}{\tau_{D}} .
$$

Using the results of the lifetime analysis for the CCP/Ag@SiO ${ }_{2} @ S i O_{2}+$ FiTC system, transfer efficiencies of 4 and 50\% were calculated for the silica and the core-shell nanoparticles, respectively. These transfer efficiency values can then be used to calculate the average donor-acceptor distance $(R)$ or the Förster distance $\left(R_{0}\right)$, that is, the distance between donor and acceptor molecules at which the energy transfer efficiency is 50\%, using this relationship [7]:

$$
E=\frac{R_{0}^{6}}{R_{0}^{6}+R^{6}} .
$$

Using a $R_{0}$ of $50 \AA$ for the CCP-fluorescein system and a transfer efficiency of $4 \%$ for the silica particles, an average distance of $50 \AA$ between fluorescein and the CCP was calculated. Since the fluorophores are covalently attached to the rigid silica shells using the same mechanism, the average donor-acceptor distance is expected to be very similar in core-shell NPs and in their corresponding silica particles. Consequently, using an $R$ equal to $50 \AA$ and a transfer efficiency of $50 \%$, a metal-enhanced $R_{0(\mathrm{M})}$ of $85 \AA$ was calculated or an increase of $\sim 70 \%$ for these multilayer coreshell NPs. These results allowed us to postulate that plasmonic coupling in the core-shell nanocomposites should allow the CCP to excite a larger number of acceptors over a given period of time and over distances exceeding the natural range of FRET measurements.

\section{Applications}

The MEF- and ME-FRET-capable multilayer nanoparticles described in this report offer significant advantages for the development of novel molecular sensing schemes. For example, core-shell multilayer dye-doped acceptor NPs grafted with short single-stranded DNA (ssDNA) probes and complexed with a cationic conjugated polymer (CCP) were demonstrated as a sensitive DNA sensor (Figure 15) [19]. This approach exploits the chromism of the CCP, that is, intensity and spectral changes in absorption and luminescence caused by conformational changes in the CCP's conjugated backbone upon electrostatic binding to DNA strands. When the CCP is added to the probe-grafted NPs, it binds electrostatically to negatively charged ssDNA probes and adopts a planar and highly conjugated form in which fluorescence is effectively quenched (Figure 15(a)). The presence of complementary DNA targets leads to hybridization of the latter with the probes, forcing the polymer to rearrange itself around the DNA double helix and revert to a nonplanar, fluorescent conformation, whereupon excitation of the polymer donor at $410 \mathrm{~nm}$ results in sensitized emission (via resonant energy 


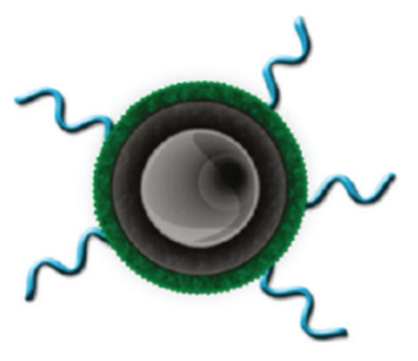

Probe-grafted NPs

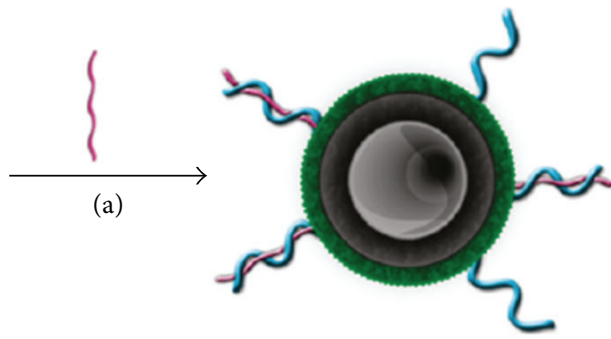

Target-ready NPs

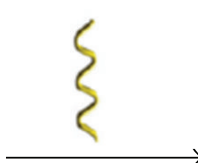

(b)

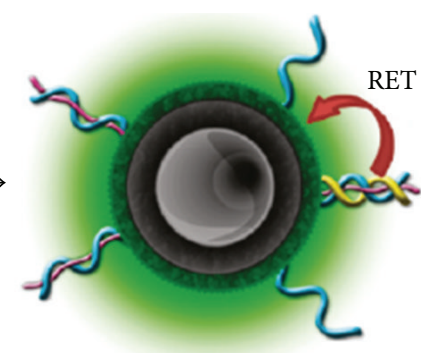

Target-activated fluorescent NPs

FIGURE 15: Principle of DNA sensing using fluorescent multilayer core-shell NPs: (a) target-ready NPs are prepared by complexing ssDNA probe-grafted NPs with a polymeric hybridization transducer; (b) hybridization of target DNA with ssDNA probes activates the polymer transducer as energy donor toward dye-doped silica shell and excitation at $410 \mathrm{~nm}$ generates fluorescence emission by acceptor molecules at $550 \mathrm{~nm}$. (Reprinted with permission from [19], Copyright 2011 American Chemical Society).

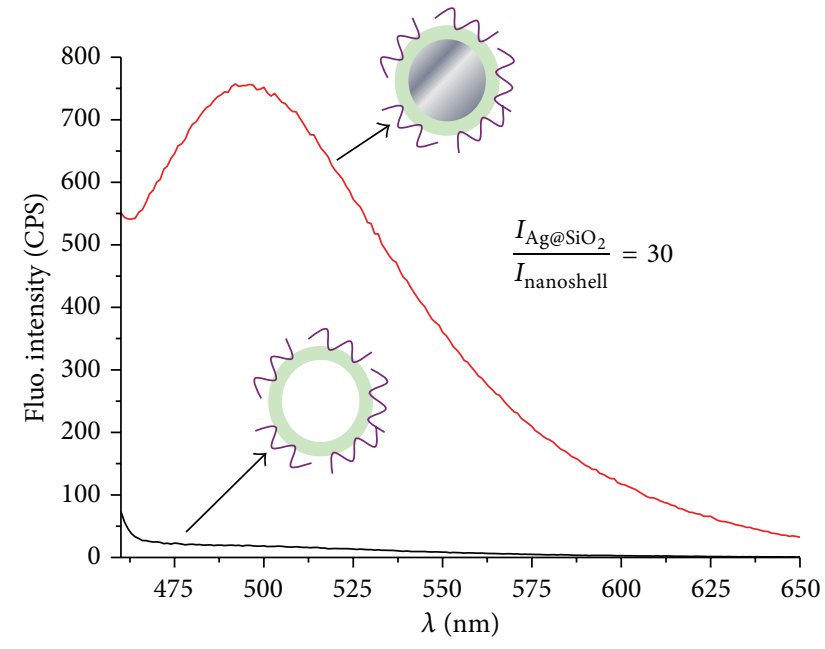

FIGURE 16: Steady-state fluorescence spectra for CCP adsorbed on $\mathrm{Ag} @ \mathrm{SiO}_{2} \mathrm{NPs}_{\text {and }}$ on coreless nanoshells. The excitation wavelength was $400 \mathrm{~nm}$. (Reprinted with permission from [41], Copyright 2011 American Chemical Society).

transfer) from the polymer-DNA complexes to the acceptor molecules (eosin) immobilized in the outer silica layer of the NPs (Figure 15(b)). In this design, the luminescence of both polymer and eosin molecules is strongly enhanced in proximity to the silver core (e.g., Figures $8(a)$ and 16). Also, the capacity of the polymer as energy donor is increased by strong near-field interactions in this plasmonic-enhanced FRET system and results in faster transfer rate and enhanced FRET efficiency and range.

Since the polymer exists in its quenched planar conformation in the target-ready NPs, the latter are not fluorescent when observed by imaging flow cytometry (IFC) and excited near the nominal excitation wavelength of the polymer donor. However, they were easily detectable when excited directly at the nominal excitation wavelength of the eosin acceptor at $488 \mathrm{~nm}$ and, upon the addition of complementary oligonucleotides to the target-ready NPs, excitation of CCP resulted in the appearance of fluorescent objects that were on average significantly larger than on images recorded from probe-grafted NPs before addition of the CCP [19].

The formation of these larger objects, presumed to be aggregates of target-ready NPs, is believed to arise from the partial neutralization of negative surface charges on probegrafted NPs following the addition of the CPP transducer, and hybridization of target molecules onto these aggregates then leads to readily detectable changes in luminosity (Figure 17). Such nanoparticle networks could allow collective interactions between NPs, thereby enhancing plasmonic coupling and local electric field intensities between them and amplifying the overall optical signal generated by DNA hybridization events.

As illustrated in Figure 18, a linear correlation was found between fluorescence signal intensity and target analyte concentration, and specific detection of a few thousand copies of complementary oligonucleotides in a few minutes was demonstrated with this molecular sensing system. In the case of 20 mer target oligonucleotides, the limit of detection was calculated as $\sim 1 \times 10^{4}$ molecules, or $2 \times 10^{-20} \mathrm{~mol}$ in the $20 \mu \mathrm{L}$ sample aliquot measured by IFC, or $8 \times 10^{-16} \mathrm{M}$. In comparison with perfectly matched target, the presence of a large excess $(100 x)$ of sequences having two mismatches led only to a slight increase in luminescence. This high selectivity toward perfectly matched targets is granted by the very high stringency used during the detection step, that is, in pure water at $60^{\circ} \mathrm{C}$; in such conditions, hybridization essentially occurs only with the ssDNA probes electrostatically bound to the cationic polythiophene chains, which are the only counter-ion available for the negative charges of the phosphate moieties of the DNA helix. The signal measured with complementary targets was found to be linear up to $\sim 10^{-12} \mathrm{M}\left(\sim 6 \times 10^{5}\right.$ copies $\left./ \mu \mathrm{L}\right)$, but to decrease at higher concentrations. Considering the large number of probes available on each nanoparticle on average ( 1500/NP, or $\sim 6 \times$ $10^{8} / \mu \mathrm{L}$ ), the particle binding capacity is not expected to be a limiting factor. Rather, the departure from linearity observed at higher concentration might result from the disruption of NP aggregates due to increasing electrostatic repulsions within them.

The sensitive response of NP aggregates to the addition of complementary targets is incumbent on a combination 

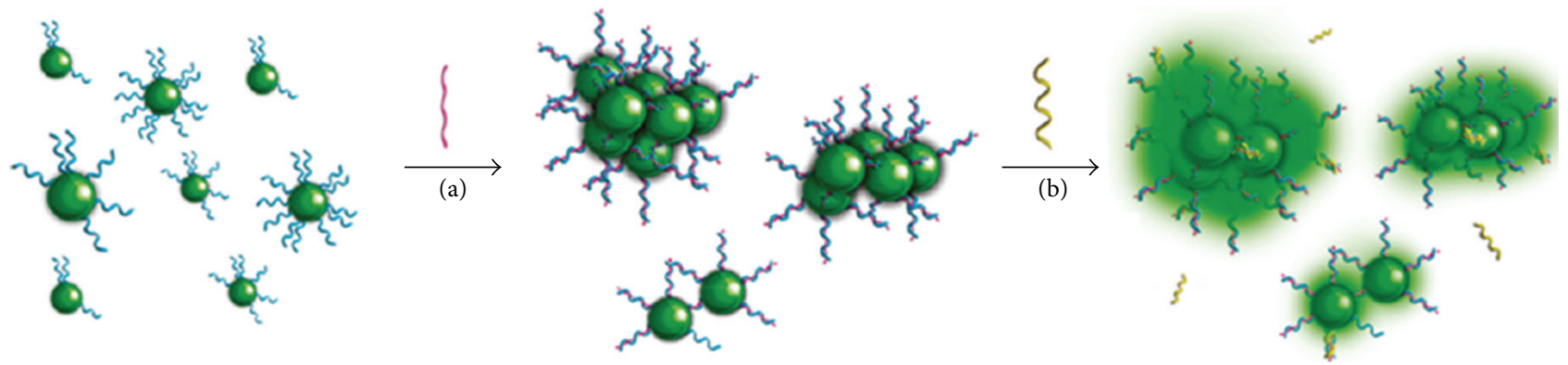

FIGURE 17: DNA detection using polymer-induced particle networks: (a) target-ready NP aggregate following partial charge neutralization by cationic polymer; (b) hybridization of target oligonucleotides activates polymer transducer as RET donor toward dye-doped silica shell; excitation at $410 \mathrm{~nm}$ generates fluorescence by acceptor molecules at $550 \mathrm{~nm}$. (Reprinted with permission from [19], Copyright 2011 American Chemical Society).

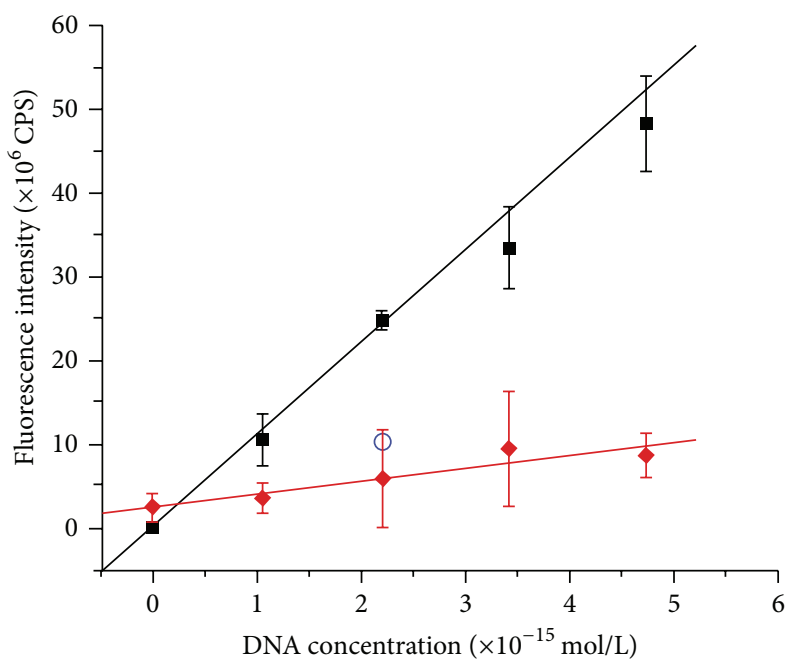

(a)

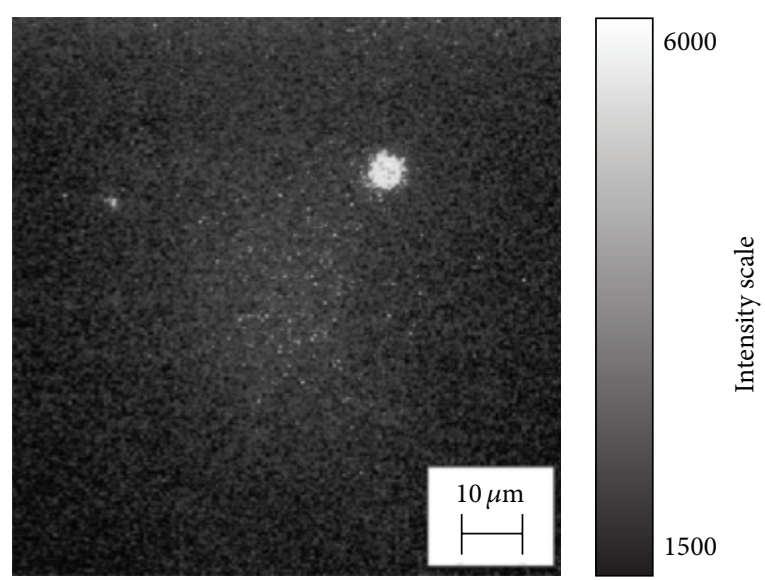

(b)

FIGURE 18: (a) Calibration curves for 20 mer single-stranded DNA targets measured with target-ready NPs: perfectly matched (squares) and two-mismatch (diamonds) targets. The open circle indicates the signal recorded for a 100x excess of the two-mismatch sequence. Limit of detection for perfectly matched targets is $1 \times 10^{4}$ molecules or $20 \mathrm{zmol}$ in a volume of $20 \mu \mathrm{L}$. (b) Example of fluorescence image recorded by IFC. (Reprinted with permission from [19], Copyright 2011 American Chemical Society; [43], Copyright 2013 The Royal Society of Chemistry).

of several elements. The very structure of the probe-labeled core-shell NPs and the resulting polymer-induced aggregates maximize the proximity between donors and acceptors that is required for optimal RET. Moreover, strong plasmonic coupling between metal cores is responsible for the enhanced luminosity of fluorescent species as well as the increased range and efficiency of RET, favoring the excitation of several acceptors by each polymer donor throughout the aggregate system. Finally, each hybridization event recognized by the CCP transducer is signaled by a large number of excited reporters following the efficient plasmon-mediated energy transfer between the target-activated polymer transducer and the numerous fluorophores located in the self-assembled core-shell aggregates. This combination of events is what allows the sensitive and rapid detection of target nucleic acids at low femtomolar $\left(10^{-15} \mathrm{M}\right)$ concentrations. This detection sensitivity, while being somewhat higher than the widely used enzymatic amplification (PCR, for Polymerase Chain Reaction) techniques, is nevertheless commensurate with DNA levels typically extracted from a few microliters of blood.

This core-shell plasmonic architecture was therefore implemented for the direct detection of a specific gene (SRY or "sex-determining region of $\mathrm{Y}$ ") from unamplified human genomic DNA [44].Ag@SiO ${ }_{2} @ \mathrm{SiO}_{2}+$ EiTC particles functionalized with 22 mer probes specific to a region of the SRY gene were complexed with the CCP transducer and allowed to hybridize with purified human DNA extracted from blood samples during $10 \mathrm{~min}$ at $55^{\circ} \mathrm{C}$. The results were correlated with classic PCR amplification and gel electrophoresis (Figure 19). The nanobiosensor allowed the correct identification of the $S R Y$ gene in 9 out of 10 cases, with the results available less than 10 minutes after mixing. Despite the low amount of DNA available, a clear signal contrast 


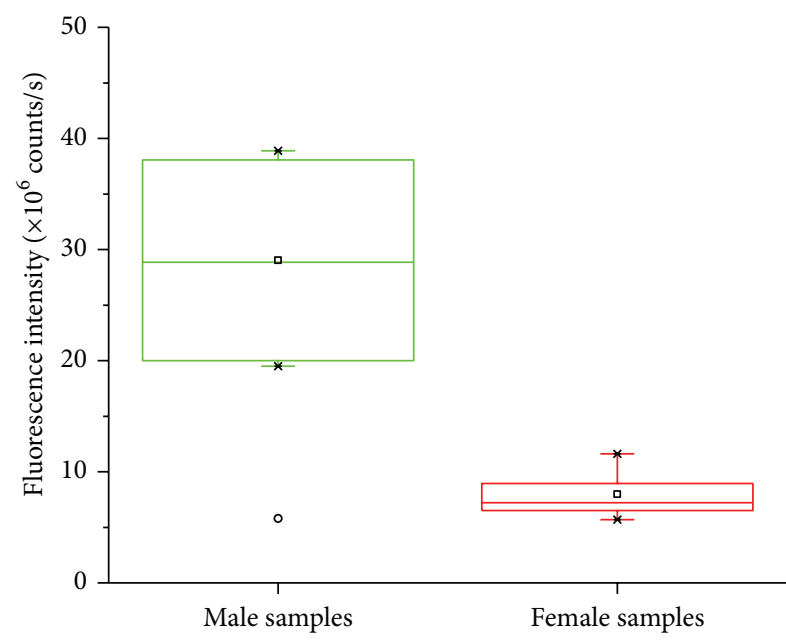

FIGURE 19: SRY genotyping results for 10 unknown genomic DNA samples extracted from human blood samples. Results obtained with nanobiosensor were correlated with PCR analysis. Five samples were female (negative) and the five others were male (positive). 9 out of 10 samples were correctly identified with the nanobiosensor. The 10th sample (false negative) is represented as an open circle. (Reprinted with permission from [44], Copyright 2013 The Royal Society of Chemistry).

was found between the positive and negative samples, a consequence both of the very high detection sensitivity of the nanobiosensor and of the ability of the polymer transducer to promote hybridization under very stringent conditions (i.e., at $55^{\circ} \mathrm{C}$ and in pure water, without other counter-ions to neutralize the negative charges of the DNA backbone phosphates). This detection contrast is remarkable, considering the diversity of the human genome, which contains multiple one- or two-base mismatched sequences with the SRY target.

\section{Summary and Outlook}

In this paper, we have presented recent advances in our efforts to design nanoobjects with well-defined tridimensional geometry that are capable of metal-enhanced fluorescence. These multishell metal/silica nanoparticles combine the properties of more conventional dye-doped silica NPs (i.e., high optical detectability, large spectral coverage, excellent chemical and physical stability, low toxicity, high solubility in water, and easy conjugation to target biomolecules) together with the enhanced luminescence intensity, excitation crosssection, and photostability resulting from the plasmonic interactions occurring in these nanostructures. Moreover, RET efficiency, transfer rate, and range are all significantly improved by the core-shell configuration. Interestingly, in comparison with metal-enhanced experiments performed using planar substrates such as silver island films, this " $3-\mathrm{D}$ " MEF detection platform, thanks to the greater mobility of the nanocomposites, allows interaction with a greater fraction of the sample volume in biosensing and bioimaging applications and has been used successfully for the ultrasensitive and sequence-specific sensing of minute amounts of DNA without any labeling or amplification of the nucleic acids. These MEF-capable, core-shell nanocomposites possess many of the features required of an ideal fluorescent probe and, despite the exacting synthesis procedures required to produce NPs with highly uniform geometrical features of a few nanometers in size, a growing number of laboratories are now pursuing their development, prompted by the enviable properties of these nanocomposites which allow us to envision a myriad of applications in cell and cancer biology, nanomedecine, and medical diagnostics.

Even though multiple applications become possible or perfected with this plasmonic multilayer core-shell architecture, one might expect future research to bring new developments on the table to optimize and refine the system. As illustrated in Figure 17, our results show an amplification in the luminescence signal after formation of aggregates [19]. Various studies have used simulated and experimental results to prove the relation of the proximity between metallic cores and their plasmonic coupling [67]. Better understanding of such propagation and its influence on fluorophores could eventually lead to the formation of controlled nanoaggregates exhibiting an enhanced fluorescence intensity. As described previously, in our case, double-strand DNA hybridization with a cationic polymer resulted in a minimization of electrostatic repulsions between the bioprobes in suspension. Under precise conditions, it might become possible to form different type of networks favoring and modulating the plasmonic coupling. Such nanoorganized sensors could potentially reach impressively low limits of detection, leading to better diagnosis in many analytical fields.

However, much as the effects of MEF on organic molecules have been widely studied, on nontraditional fluorescence emitters, they have yet to be applied. Upconversion or atomic emission in lanthanide ions [68] and quantum dots (QDs) [69, 70] are known to be influenced by the presence of plasmonic modules, whether by quenching or enhancement from metallic nanoparticles or surfaces. Moreover, their optical properties are in many ways superior to most dyes: better excitation and emission tunability, larger Stokes shift, minimal autoabsorption, and distinctive fluorescence lifetime, to name a few. Therefore, physical studies of plasmonic mechanisms on such attractive fluorophores could greatly improve our comprehension of both MEF and ME-FRET process and also diversify the number of potential nanobioprobes usable in the foreseeable future. Indeed, since these nanoparticle-based emitters can be functionalized in a similar manner to core-shell NPs, one could probably imagine a way to create nanoaggregates incorporating both to form a well-ordered network, thus propagating an enhancing plasmonic resonance throughout the corpuscle.

The main inconvenient crippling the application of nontraditional fluorophores in medical fields is the toxicity incumbent with their composition. To be sure, uses of most plasmonic metals (silver, aluminium, indium, etc.) in biological environments are known to come with certain cytotoxic effects, the importance of which may vary [71]. Consequently, some attention should be put in the development of protection methods for the metallic cores, with 
better efficiency than porous silica. For example, chemical surface passivation - as in the case of indium NPs [42]could be adapted to other compositions to serve both as a nonplasmonic spacer and a stable protective shell for the core. Many variations of the polymeric condensation of silica on metallic NPs exist, differing from the traditional Stöber methodology [72]. The well-known chemistry of silica offers many advantages but, in the case of core-shell fluorescent nanosensors, the option of covalent fluorophore doping is particularly significant. However, because the surface plasmon resonance phenomenon is dependent on the local refraction index, variations in the porosity of the dielectric layer might have important effects on MEF properties of the dye molecules entrapped inside. As such, this problematic could prove to be a promising challenge and its resolution would lead to even more analytical applications, mostly by opening the door toward in situ detections of cellular environments.

\section{Conflict of Interests}

The authors declare that there is no conflict of interests regarding the publication of this paper.

\section{Acknowledgments}

The authors are grateful to the National Sciences and Engineering Research Council of Canada, the Canada Foundation for Innovation, the Fonds pour la Recherche du QuébecNature et Technologies, Héma-Québec, and the Canadian Blood Services for financial support. The authors also thank Marie-France Champigny of the "Institut universitaire en santé mentale de Québec" and Marc Choquette of the "Laboratoire de Microanalyse de l'Université Laval" for help with TEM sample preparation and characterization. The contribution of Professor Mario Leclerc for the synthesis of the polymer transducer is also acknowledged.

\section{References}

[1] M. Hof, R. Hutterer, and V. Fidler, Eds., Fluorescence Spectroscopy in Biology: Advances Methods and Their Applications to Membranes, Proteins, DNA, and Cells, Springer, New York, NY, USA, 2005.

[2] D. Toomre and J. Bewersdorf, "A new wave of cellular imaging," Annual Review of Cell and Developmental Biology, vol. 26, pp. 285-314, 2010.

[3] T. A. Brettell, J. M. Butler, and J. R. Almirall, "Forensic science," Analytical Chemistry, vol. 81, no. 12, pp. 4695-4711, 2009.

[4] K. Doré, S. Dubus, H.-A. Ho et al., "Fluorescent polymeric transducer for the rapid, simple, and specific detection of nucleic acids at the zeptomole level," Journal of the American Chemical Society, vol. 126, no. 13, pp. 4240-4244, 2004.

[5] G. Palacios, P.-L. Quan, O. J. Jabado et al., "Panmicrobial oligonucleotide array for diagnosis of infectious diseases," Emerging Infectious Diseases, vol. 13, no. 1, pp. 73-81, 2007.

[6] W. P. Gati, "Flow cytometry," in Handbook of Neurochemistry and Molecular Neurobiology: Practical Neurochemistry Methods, pp. 305-321, 3rd edition, 2007.
[7] J. R. Lakowicz, Principles of Fluorescence Spectroscopy, Plenum Publishing Corporation, New York, NY, USA, 2nd edition, 1999.

[8] W. L. Barnes, "Fluorescence near interfaces: the role of photonic mode density," Journal of Modern Optics, vol. 45, no. 4, pp. 661699, 1998.

[9] J. R. Lakowicz, J. Malicka, I. Gryczynski, Z. Gryczynski, and C. D. Geddes, "Radiative decay engineering: the role of photonic mode density in biotechnology," Journal of Physics D: Applied Physics, vol. 36, no. 14, pp. R240-R249, 2003.

[10] C. D. Geddes, K. Aslan, I. Gryczynski, and J. R. Lakowicz, "Metal-enhanced fluorescence sensing," in Fluorescence Sensors and Biosensors, pp. 121-181, 2006.

[11] C. D. Geddes and J. R. Lakowicz, Eds., Radiative Decay Engineering, vol. 8 of Topics in Fluorescence Spectroscopy, Springer, New York, NY, USA, 2005.

[12] C. McDonagh, O. Stranik, R. Nooney, and B. D. MacCraith, "Optimisation of plasmonic enhancement of fluorescence for optical biosensor applications," in Metal-Enhanced Fluorescence, pp. 139-160, John Wiley \& Sons, New York, NY, USA, 2010.

[13] R. Nooney, A. Clifford, X. Leguevel, O. Stranik, C. McDonagh, and B. D. MacCraith, "Enhancing the analytical performance of immunoassays that employ metal-enhanced fluorescence," Analytical and Bioanalytical Chemistry, vol. 396, no. 3, pp. 11271134, 2010.

[14] C. McDonagh, O. Stranik, R. Nooney, and B. D. MacCraith, "Nanoparticle strategies for enhancing the sensitivity of fluorescence-based biochips," Nanomedicine, vol. 4, no. 6, pp. 645-656, 2009.

[15] Y. Fu, J. Zhang, and J. R. Lakowicz, "Metallic-nanostructureenhanced fluorescence of single flavin cofactor and single flavoenzyme molecules," Journal of Physical Chemistry C, vol. 115, no. 15, pp. 7202-7208, 2011.

[16] J. Zhang, Y. Fu, and J. R. Lakowicz, "Fluorescent metal nanoshells: lifetime-tunable molecular probes in fluorescent cell imaging," Journal of Physical Chemistry C, vol. 115, no. 15, pp. 7255-7260, 2011.

[17] J. Zhang, Y. Fu, Y. Mei, F. Jiang, and J. R. Lakowicz, "Fluorescent metal nanoshell probe to detect single mirna in lung cancer cell," Analytical Chemistry, vol. 82, no. 11, pp. 4464-4471, 2010.

[18] R.-Y. He, Y.-D. Su, K.-C. Cho et al., "Surface plasmon-enhanced two-photon fluorescence microscopy for live cell membrane imaging," Optics Express, vol. 17, no. 8, pp. 5987-5997, 2009.

[19] D. Brouard, M. L. Viger, A. G. Bracamonte, and D. Boudreau, "Label-free biosensing based on multilayer fluorescent nanocomposites and a cationic polymeric transducer," ACS Nano, vol. 5, no. 3, pp. 1888-1896, 2011.

[20] K. Golberg, A. Elbaz, Y. Zhang, A. I. Dragan, R. Marks, and C. D. Geddes, "Mixed-metal substrates for applications in metalenhanced fluorescence," Journal of Materials Chemistry, vol. 21, no. 17, pp. 6179-6185, 2011.

[21] N. Bondre, Y. Zhang, and C. D. Geddes, "Metal-enhanced fluorescence based calcium detection: greater than 100-fold increase in signal/noise using Fluo-3 or Fluo-4 and silver nanostructures," Sensors and Actuators, B: Chemical, vol. 152, no. 1, pp. 82-87, 2011.

[22] Y. Zhang, A. Dragan, and C. D. Geddes, "Wavelength dependence of metal-enhanced fluorescence," Journal of Physical Chemistry C, vol. 113, no. 28, pp. 12095-12100, 2009.

[23] K. Aslan, J. R. Lakowicz, and C. D. Geddes, "Metal-enhanced fluorescence using anisotropic silver nanostructures: critical 
progress to date," Analytical and Bioanalytical Chemistry, vol. 382, no. 4, pp. 926-933, 2005.

[24] C. D. Geddes, A. Parfenov, D. Roll, I. Gryczynski, J. Malicka, and J. R. Lakowicz, "Roughened silver electrodes for use in metalenhanced fluorescence," Spectrochimica Acta A: Molecular and Biomolecular Spectroscopy, vol. 60, no. 8-9, pp. 1977-1983, 2004.

[25] K. Aslan, Z. Leonenko, J. R. Lakowicz, and C. D. Geddes, "Fast and slow deposition of silver nanorods on planar surfaces: application to metal-enhanced fluorescence," Journal of Physical Chemistry B, vol. 109, no. 8, pp. 3157-3162, 2005.

[26] K. Aslan, R. Badugu, J. R. Lakowicz, and C. D. Geddes, "Metal-enhanced fluorescence from plastic substrates," Journal of Fluorescence, vol. 15, no. 2, pp. 99-104, 2005.

[27] K. Aslan, J. Huang, G. M. Wilson, and C. D. Geddes, "Metalenhanced fluorescence-based RNA sensing," Journal of the American Chemical Society, vol. 128, no. 13, pp. 4206-4207, 2006.

[28] C. D. Geddes, A. Parfenov, D. Roll, J. Fang, and J. R. Lakowicz, "Electrochemical and laser deposition of silver for use in metalenhanced fluorescence," Langmuir, vol. 19, no. 15, pp. 6236-6241, 2003.

[29] F. Xie, M. S. Baker, and E. M. Goldys, "Homogeneous silvercoated nanoparticle substrates for enhanced fluorescence detection," Journal of Physical Chemistry B, vol. 110, no. 46, pp. 2308523091, 2006.

[30] R. Bardhan, N. K. Grady, J. R. Cole, A. Joshi, and N. J. Halas, "Fluorescence enhancement by au nanostructures: nanoshells and nanorods," ACS Nano, vol. 3, no. 3, pp. 744-752, 2009.

[31] O. G. Tovmachenko, C. Graf, D. J. van den Heuvel, A. van Blaaderen, and H. C. Gerritsen, "Fluorescence enhancement by metal-core/silica-shell nanoparticles," Advanced Materials, vol. 18, no. 1, pp. 91-95, 2006.

[32] J. Zhang, Y. Fu, M. H. Chowdhury, and J. R. Lakowicz, "Singlemolecule studies on fluorescently labeled silver particles: effects of particle size," Journal of Physical Chemistry C, vol. 112, no. 1, pp. 18-26, 2008.

[33] K. Aslan, M. Wu, J. R. Lakowicz, and C. D. Geddes, "Fluorescent core-shell Ag@SiO 2 nanocomposites for metal-enhanced fluorescence and single nanoparticle sensing platforms," Journal of the American Chemical Society, vol. 129, no. 6, pp. 1524-1525, 2007.

[34] M. Martini, P. Perriat, M. Montagna et al., "How gold particles suppress concentration quenching of fluorophores encapsulated in silica beads," Journal of Physical Chemistry C, vol. 113, no. 41, pp. 17669-17677, 2009.

[35] O. Stranik, R. Nooney, C. McDonagh, and B. D. MacCraith, "Plasmonic enhancement using core-shell nanoparticles," in Opto-Ireland 2005: Nanotechnology and Nanophotonics, vol. 5824 of Proceedings of SPIE, pp. 79-85, Dublin, Ireland, April 2005.

[36] J. Zhang, Y. Fu, and J. R. Lakowicz, "Enhanced Förster resonance energy transfer (FRET) on a single metal particle," Journal of Physical Chemistry C, vol. 111, no. 1, pp. 50-56, 2007.

[37] D. Cheng and Q.-H. Xu, "Separation distance dependent fluorescence enhancement of fluorescein isothiocyanate by silver nanoparticles," Chemical Communications, no. 3, pp. 248-250, 2007.

[38] L. Guo, A. Guan, X. Lin, C. Zhang, and G. Chen, "Preparation of a new core-shell Ag@SiO ${ }_{2}$ nanocomposite and its application for fluorescence enhancement," Talanta, vol. 82, no. 5, pp. 16961700, 2010.
[39] M. Lessard-Viger, M. Rioux, L. Rainville, and D. Boudreau, "FRET enhancement in multilayer core-shell nanoparticles," Nano Letters, vol. 9, no. 8, pp. 3066-3071, 2009.

[40] M. L. Viger, L. S. Live, O. D. Therrien, and D. Boudreau, "Reduction of self-quenching in fluorescent silica-coated silver nanoparticles," Plasmonics, vol. 3, no. 1, pp. 33-40, 2008.

[41] M. L.-Viger, D. Brouard, and D. Boudreau, "Plasmon-enhanced resonance energy transfer from a conjugated polymer to fluorescent multilayer core-shell nanoparticles: a photophysical study," Journal of Physical Chemistry C, vol. 115, no. 7, pp. 29742981, 2011.

[42] F. Magnan, J. Gagnon, F. G. Fontaine, and D. Boudreau, "Indium@silica core-shell nanoparticles as plasmonic enhancers of molecular luminescence in the UV region," Chemical Communications, vol. 49, no. 81, pp. 9299-9301, 2013.

[43] J. R. Lakowicz, J. Malicka, S. D’Auria, and I. Gryczynski, "Release of the self-quenching of fluorescence near silver metallic surfaces," Analytical Biochemistry, vol. 320, no. 1, pp. 13-20, 2003.

[44] D. Brouard, O. Ratelle, A. G. Bracamonte, M. St-Louis, and D. Boudreau, "Direct molecular detection of SRY gene from unamplified genomic DNA by metal-enhanced fluorescence and FRET," Analytical Methods, vol. 5, no. 24, pp. 6896-6899, 2013.

[45] S. Liu, Z. Zhang, and M. Han, "Gram-scale synthesis and biofunctionalization of silica-coated silver nanoparticles for fast colorimetric DNA detection," Analytical Chemistry, vol. 77, no. 8, pp. 2595-2600, 2005.

[46] X. Ji, X. Song, J. Li, Y. Bai, W. Yang, and X. Peng, "Size control of gold nanocrystals in citrate reduction: the third role of citrate," Journal of the American Chemical Society, vol. 129, no. 45, pp. 13939-13948, 2007.

[47] J. Turkevich, P. C. Stevenson, and J. Hillier, "A study of the nucleation and growth processes in the synthesis of colloidal gold," Discussions of the Faraday Society, vol. 11, pp. 55-75, 1951.

[48] X. Dong, X. Ji, H. Wu, L. Zhao, J. Li, and W. Yang, "Shape control of silver nanoparticles by stepwise citrate reduction," Journal of Physical Chemistry C, vol. 113, no. 16, pp. 6573-6576, 2009.

[49] L. Rainville, M. C. Dorais, and D. Boudreau, "Controlled synthesis of low polydispersity $\mathrm{Ag} @ \mathrm{SiO}_{2}$ core-shell nanoparticles for use in plasmonic applications," RSC Advances, vol. 3, no. 33, pp. 13953-13960, 2013.

[50] M. Brust, M. Walker, D. Bethell, D. J. Schiffrin, and R. Whyman, "Synthesis of thiol-derivatised gold nanoparticles in a twophase liquid-liquid system," Journal of the Chemical Society, Chemical Communications, no. 7, pp. 801-802, 1994.

[51] C. J. Murphy, T. K. Sau, A. M. Gole et al., "Anisotropic metal nanoparticles: synthesis, assembly, and optical applications," Journal of Physical Chemistry B, vol. 109, no. 29, pp. 13857-13870, 2005.

[52] T. H. Lim, B. Ingham, K. H. Kamarudin, P. G. Etchegoin, and R. D. Tilley, "Solution synthesis of monodisperse indium nanoparticles and highly faceted indium polyhedra," Crystal Growth and Design, vol. 10, no. 9, pp. 3854-3858, 2010.

[53] A. Kumar, H. M. Joshi, A. B. Mandale et al., "Phase transfer of platinum nanoparticles from aqueous to organic solutions using fatty amine molecules," Journal of Chemical Sciences, vol. 116, no. 5, pp. 293-300, 2004.

[54] L. M. Liz-Marzán and A. P. Philipse, "Stable hydrosols of metallic and bimetallic nanoparticles immobilized on imogolite fibers," Journal of Physical Chemistry, vol. 99, no. 41, pp. 1512015128, 1995. 
[55] Y. Chen, K. Munechika, and D. S. Ginger, "Dependence of fluorescence intensity on the spectral overlap between fluorophores and plasmon resonant single silver nanoparticles," Nano Letters, vol. 7, no. 3, pp. 690-696, 2007.

[56] W. Stöber, A. Fink, and E. Bohn, "Controlled growth of monodisperse silica spheres in the micron size range," Journal of Colloid and Interface Science, vol. 26, no. 1, pp. 62-69, 1968.

[57] T. Ung, L. M. Liz-Marzán, and P. Mulvaney, "Controlled method for silica coating of silver colloids. Influence of coating on the rate of chemical reactions," Langmuir, vol. 14, no. 14, pp. 37403748, 1998.

[58] T. Li, J. Moon, A. A. Morrone, J. J. Mecholsky, D. R. Talham, and J. H. Adair, "Preparation of $\mathrm{Ag} / \mathrm{SiO}_{2}$ nanosize composites by a reverse micelle and sol-gel technique," Langmuir, vol. 15, no. 13, pp. 4328-4334, 1999.

[59] M. B. Francis, "Updating the bioconjugation catalog," in Bioconjugate Techniques, G. T. Hermanson, Ed., vol. 4, p. 717, Academic Press, New York, NY, USA, 2nd edition, 2008.

[60] A. Wokaun, H.-P. Lutz, A. P. King, U. P. Wild, and R. R. Ernst, "Energy transfer in surface enhanced luminescence," The Journal of Chemical Physics, vol. 79, no. 1, pp. 509-514, 1983.

[61] M. A. Markowitz, P. E. Schoen, P. Kust, and B. P. Gaber, "Surface acidity and basicity of functionalized silica particles," Colloids and Surfaces A: Physicochemical and Engineering Aspects, vol. 150, no. 1-3, pp. 85-94, 1999.

[62] E. Sutter and P. Sutter, "Size-dependent room temperature oxidation of in nanoparticles," Journal of Physical Chemistry C, vol. 116, no. 38, pp. 20574-20578, 2012.

[63] J. Malicka, I. Gryczynski, J. Fang, J. Kusba, and J. R. Lakowicz, "Photostability of Cy3 and Cy5-Labeled DNA in the presence of metallic silver particles," Journal of Fluorescence, vol. 12, no. 3-4, pp. 439-447, 2002.

[64] A. Imhof, M. Megens, J. J. Engelberts, D. T. N. de Lang, R. Sprik, and W. L. Vos, "Spectroscopy of fluorescein (FITC) dyed colloidal silica spheres," Journal of Physical Chemistry B, vol. 103, no. 9, pp. 1408-1415, 1999.

[65] J. Zhang, Y. Fu, M. H. Chowdhury, and J. R. Lakowicz, "Enhanced Förster resonance energy transfer on single metal particle. 2: dependence on donor-acceptor separation distance, particle size, and distance from metal surface," Journal of Physical Chemistry C, vol. 111, no. 32, pp. 11784-11792, 2007.

[66] F. Reil, U. Hohenester, J. R. Krenn, and A. Leitner, "Förster-type resonant energy transfer influenced by metal nanoparticles," Nano Letters, vol. 8, no. 12, pp. 4128-4133, 2008.

[67] N. J. Halas, S. Lal, W.-S. Chang, S. Link, and P. Nordlander, "Plasmons in strongly coupled metallic nanostructures," Chemical Reviews, vol. 111, no. 6, pp. 3913-3961, 2011.

[68] M. Saboktakin, X. Ye, S. J. Oh et al., "Metal-enhanced upconversion luminescence tunable through metal nanoparticlenanophosphor separation," ACS Nano, vol. 6, no. 10, pp. 87588766, 2012.

[69] W. R. Algar, A. J. Tavares, and U. J. Krull, "Beyond labels: a review of the application of quantum dots as integrated components of assays, bioprobes, and biosensors utilizing optical transduction," Analytica Chimica Acta, vol. 673, no. 1, pp. 1-25, 2010.

[70] M. Lunz, X. Zhang, V. A. Gerard et al., "Effect of metal nanoparticle concentration on localized surface plasmon mediated Förster resonant energy transfer," Journal of Physical Chemistry C, vol. 116, no. 50, pp. 26529-26534, 2012.
[71] C. Leclerc and K. L. Wilkinson, "Bioaccumulation of nanosilver by Chlamydomonas reinhardtii-nanoparticle or the free ion?" Environmental Science \& Technology, vol. 48, no. 1, pp. 358-364, 2014.

[72] A. Guerrero-Martínez, J. Pérez-Juste, and L. M. Liz-Marzán, "Recent progress on silica coating of nanoparticles and related nanomaterials," Advanced Materials, vol. 22, no. 11, pp. 11821195, 2010. 

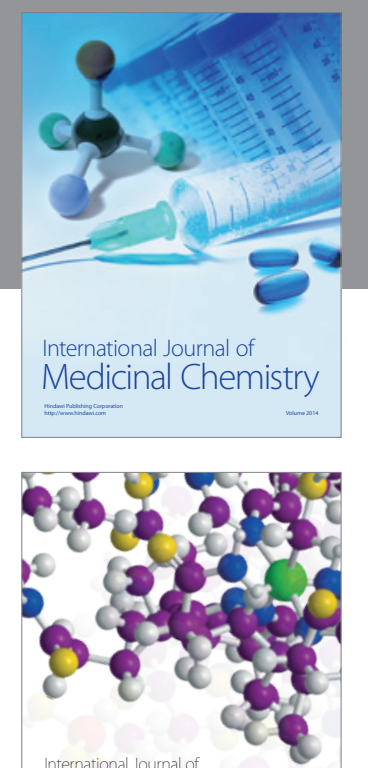

\section{Carbohydrate} Chemistry

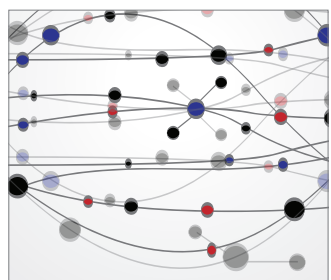

The Scientific World Journal
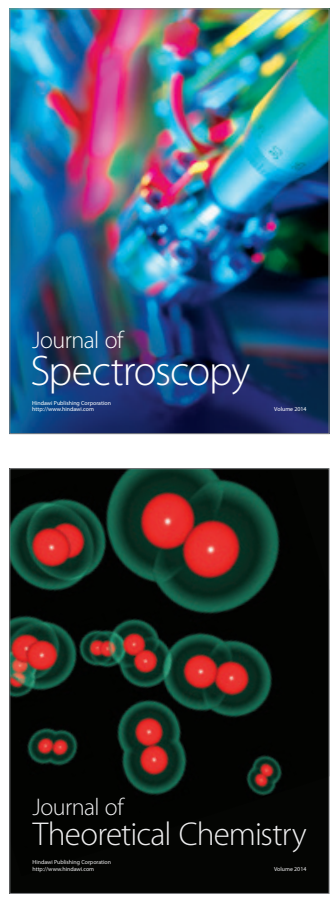
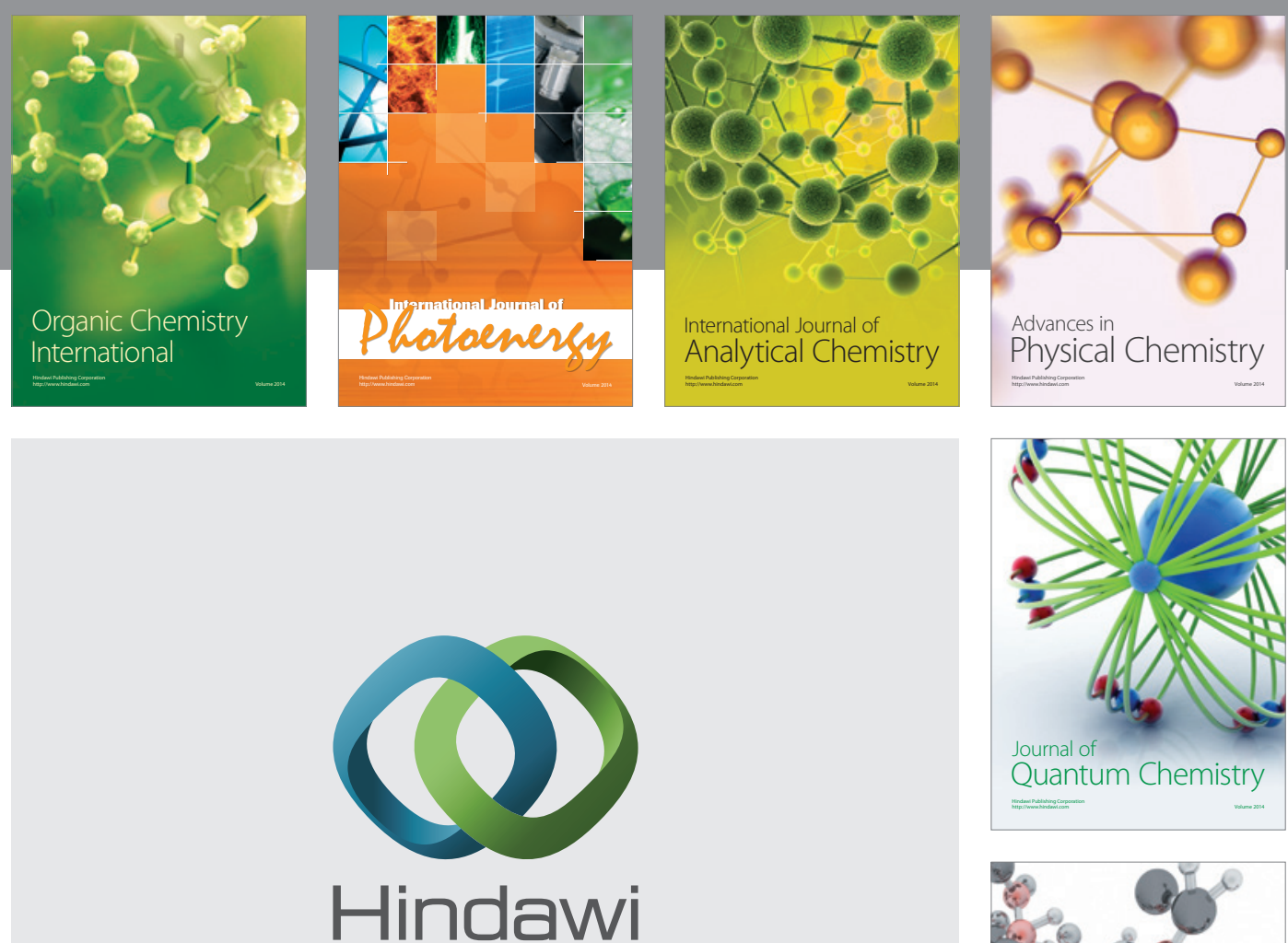

Submit your manuscripts at

http://www.hindawi.com

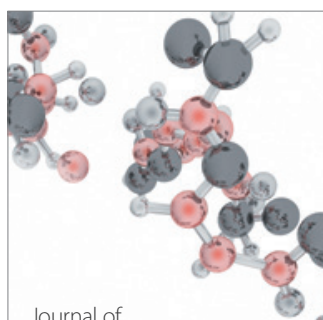

Analytical Methods

in Chemistry

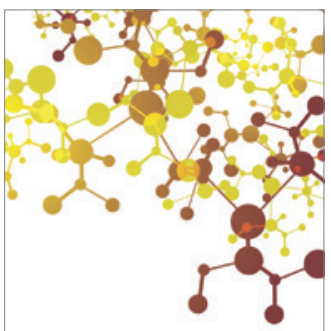

Journal of

Applied Chemistry

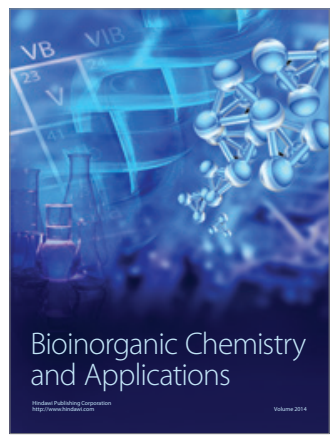

Inorganic Chemistry
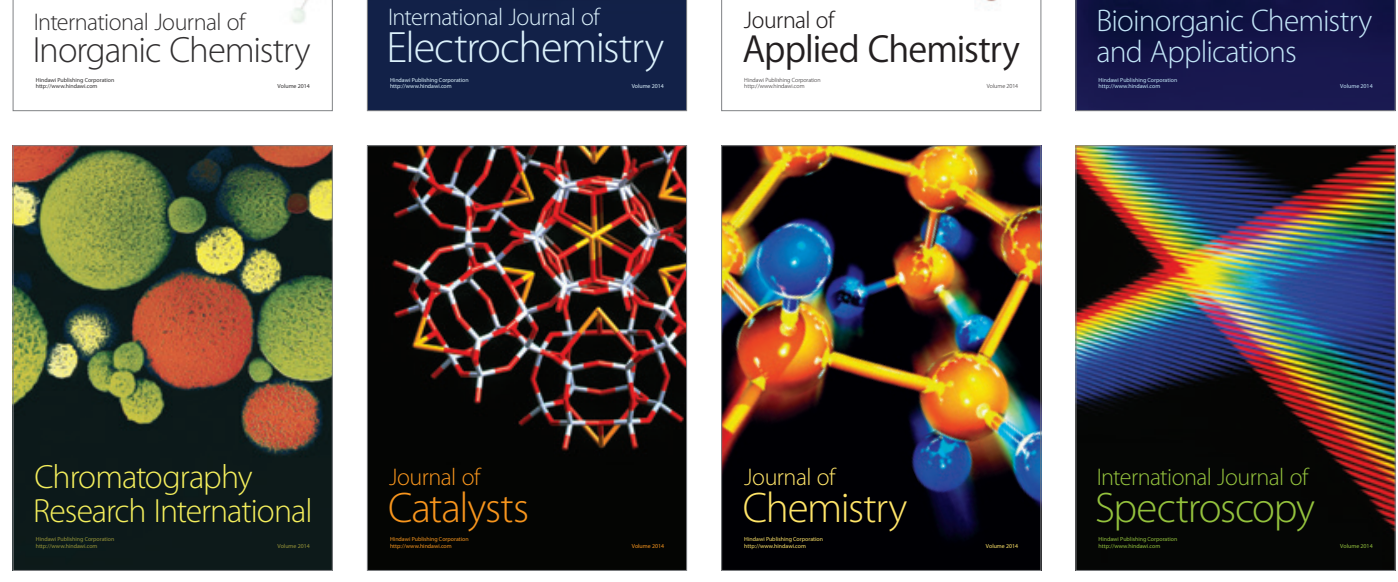